\title{
The Existence of Positive Solutions for a New Coupled System of Multiterm Singular Fractional Integrodifferential Boundary Value Problems
}

\author{
Dumitru Baleanu, ${ }^{1,2,3}$ Sayyedeh Zahra Nazemi, ${ }^{4}$ and Shahram Rezapour ${ }^{4}$ \\ ${ }^{1}$ Department of Chemical and Materials Engineering, Faculty of Engineering, King Abdulaziz University, P.O. Box 80204, \\ Jeddah 21589, Saudi Arabia \\ ${ }^{2}$ Department of Mathematics, Cankaya University, Ogretmenler Caddesi 14, Balgat, 06530 Ankara, Turkey \\ ${ }^{3}$ Institute of Space Sciences, RO 76900, Magurele-Bucharest, Romania \\ ${ }^{4}$ Department of Mathematics, Azarbaijan Shahid Madani University, Azarshahr, Tabriz 9177948974, Iran
}

Correspondence should be addressed to Dumitru Baleanu; dumitru@cankaya.edu.tr

Received 24 June 2013; Accepted 15 August 2013

Academic Editor: Juan J. Trujillo

Copyright (C) 2013 Dumitru Baleanu et al. This is an open access article distributed under the Creative Commons Attribution License, which permits unrestricted use, distribution, and reproduction in any medium, provided the original work is properly cited.

We discuss the existence of positive solutions for the coupled system of multiterm singular fractional integrodifferential boundary value problems $D_{0^{+}}^{\alpha} u(t)+f_{1}\left(t, u(t), v(t),\left(\phi_{1} u\right)(t),\left(\psi_{1} v\right)(t), D_{0^{+}}^{p} u(t), D_{0^{+}}^{\mu_{1}} v(t), D_{0^{+}}^{\mu_{2}} v(t), \ldots, D_{0^{+}}^{\mu_{m}} v(t)\right)=0, D_{0^{+}}^{\beta} v(t)+$ $f_{2}\left(t, u(t), v(t),\left(\phi_{2} u\right)(t),\left(\psi_{2} v\right)(t), D_{0^{+}}^{q} v(t), D_{0^{+}}^{\nu_{1}} u(t), D_{0^{+}}^{\nu_{2}} u(t), \ldots, D_{0^{+}}^{\nu_{m}} u(t)\right)=0, u^{(i)}(0)=0$ and $v^{(i)}(0)=0$ for all $0 \leq i \leq n-2$, $\left[D_{0^{+}}^{\delta_{1}} u(t)\right]_{t=1}=0$ for $2<\delta_{1}<n-1$ and $\alpha-\delta_{1} \geq 1,\left[D_{0^{+}}^{\delta_{2}} v(t)\right]_{t=1}=0$ for $2<\delta_{2}<n-1$ and $\beta-\delta_{2} \geq 1$, where $n \geq 4$, $n-1<\alpha, \beta<n, 0<p, q<1,1<\mu_{i}, v_{i}<2(i=1,2, \ldots, m), \gamma_{j}, \lambda_{j}:[0,1] \times[0,1] \rightarrow(0, \infty)$ are continuous functions $(j=1,2)$ and $\left(\phi_{j} u\right)(t)=\int_{0}^{t} \gamma_{j}(t, s) u(s) d s,\left(\psi_{j} v\right)(t)=\int_{0}^{t} \lambda_{j}(t, s) v(s) d s$. Here $D$ is the standard Riemann-Liouville fractional derivative, $f_{j}(j=1,2)$ is a Caratheodory function, and $f_{j}\left(t, x, y, z, w, v, u_{1}, u_{2}, \ldots, u_{m}\right)$ is singular at the value 0 of its variables.

\section{Introduction}

During the last decade, there were a lot of manuscripts on fractional differential equations (see, e.g., [1-19] and the references therein). Fractional equations have been discussed extensively as valuable tools in the modeling of many phenomena in various fields of science and engineering. Indeed, we can find numerous applications in fluid mechanics, viscoelasticity, edge detection, porous media, and electromagnetism, as well as in various other areas. For more examples and details, see $[3,13-15]$ and references therein. On the other hand, there are many works about the existence of positive solutions of fractional differential equations (see, e.g., $[1,2,6-$ $8,10,17,19]$ and the references therein).

\section{The Problem}

In this paper, we investigate the following coupled system of multiterm singular fractional integrodifferential boundary value problem:

$$
\begin{gathered}
D_{0^{+}}^{\alpha} u(t)+f_{1}\left(t, u(t), v(t),\left(\phi_{1} u\right)(t),\right. \\
\left(\psi_{1} v\right)(t), D_{0^{+}}^{p} u(t), D_{0^{+}}^{\mu_{1}} v(t), \\
\left.D_{0^{+}}^{\mu_{2}} v(t), \ldots, D_{0^{+}}^{\mu_{m}} v(t)\right)=0, \\
D_{0^{+}}^{\beta} v(t)+f_{2}\left(t, u(t), v(t),\left(\phi_{2} u\right)(t),\right.
\end{gathered}
$$




$$
\begin{gathered}
\left(\psi_{2} v\right)(t), D_{0^{+}}^{q} v(t), D_{0^{+}}^{\nu_{1}} u(t), \\
\left.D_{0^{+}}^{v_{2}} u(t), \ldots, D_{0^{+}}^{\nu_{m}} u(t)\right)=0, \\
u^{(i)}(0)=0 \quad \forall 0 \leq i \leq n-2, \\
{\left[D_{0^{+}}^{\delta_{1}} u(t)\right]_{t=1}=0 \quad \text { for } 2<\delta_{1}<n-1, \alpha-\delta_{1} \geq 1,} \\
v^{(i)}(0)=0 \quad \forall 0 \leq i \leq n-2, \\
{\left[D_{0^{+}}^{\delta_{2}} v(t)\right]_{t=1}=0 \quad \text { for } 2<\delta_{2}<n-1, \beta-\delta_{2} \geq 1,}
\end{gathered}
$$

where $n \geq 4, n-1<\alpha, \beta<n, 0<p, q<1,1<\mu_{i}, v_{i}<$ $2(i=1,2, \ldots, m), \gamma_{j}$ and $\lambda_{j}$ are positive-valued continuous functions on $[0,1] \times[0,1](j=1,2)$,

$$
\begin{aligned}
& \left(\phi_{j} u\right)(t)=\int_{0}^{t} \gamma_{j}(t, s) u(s) d s, \\
& \left(\psi_{j} v\right)(t)=\int_{0}^{t} \lambda_{j}(t, s) v(s) d s,
\end{aligned}
$$

$\gamma_{0}^{j}=\sup _{t \in[0,1]}\left|\int_{0}^{t} \gamma_{j}(t, s) d s\right|<\infty$ and $\lambda_{0}^{j}=$ $\sup _{t \in[0,1]}\left|\int_{0}^{t} \lambda_{j}(t, s) d s\right|<\infty$. Also, $f_{1}$ and $f_{2}$ satisfy the local Caratheodory condition on $[0,1] \times \mathfrak{D}\left(f_{1}, f_{2} \in\right.$ $\operatorname{Car}([0,1] \times \mathfrak{D}))$, where $\mathfrak{D}$ is a subset of $\mathbb{R}^{m+5}$ and $f_{j}\left(t, x, y, z, w, v, u_{1}, u_{2}, \ldots, u_{m}\right)$ may be singular at the value 0 of all its variables. Here, $D$ is the standard RiemannLiouville fractional derivative. In fact, a function $f$ satisfies the local Caratheodory condition on $[0,1] \times \mathfrak{D}$ whenever

(i) $f\left(\cdot ; x, y, z, w, v, u_{1}, u_{2}, \ldots, u_{m}\right):[0,1] \rightarrow \mathbb{R}$ is measurable for all $\left(x, y, z, w, v, u_{1}, u_{2}, \ldots, u_{m}\right) \in \mathfrak{D}$

(ii) $f(t ; \cdot, \cdot, \ldots, \cdot): \mathfrak{D} \rightarrow \mathbb{R}$ is continuous for almost all $t \in[0,1]$,

(iii) for each compact subset $\kappa \subset \mathfrak{D}$, there is a function $\varphi_{\kappa} \in L^{1}[0,1]$ such that

$$
\left|f\left(t ; x, y, z, w, v, u_{1}, u_{2}, \ldots, u_{m}\right)\right| \leq \varphi_{\kappa}(t),
$$

for almost all $t \in[0,1]$ and all $\left(x, y, z, w, v, u_{1}, u_{2}, \ldots, u_{m}\right) \in$ $\kappa$. The functions $u, v \in C^{2}[0,1]$ are called positive solutions of the problem (1), (2), and (3) whenever $u>0$ and $v>0$ on $(0,1], D_{0^{+}}^{\alpha} u, D_{0^{+}}^{\beta} v \in L^{1}[0,1], u, v$ satisfy boundary conditions (2), (3), and (1) holds for almost all $t \in[0,1]$. In 2010, Agarwal et al. reviewed positive solutions of the singular Dirichlet problem

$$
D_{0^{+}}^{\alpha} u(t)+f\left(t, u(t), D_{0^{+}}^{\mu} u(t)\right)=0,
$$

$u(0)=u(1)=0$, where $1<\alpha<2,0<\mu \leq \alpha-1$ and $f$ satisfies the local Caratheodory condition on $[0,1] \times(0, \infty) \times \mathbb{R}[1]$. In 2011, Staněk reviewed the singular problem

$$
D_{0^{+}}^{\alpha} u(t)+f\left(t, u(t), u^{\prime}(t), D_{0^{+}}^{\mu} u(t)\right)=0,
$$

$u(0)=0, u^{\prime}(0)=u^{\prime}(1)=0$, where $2<\alpha<3,0<\mu<1$ and $f$ satisfies the local Caratheodory condition on $[0,1] \times \mathfrak{D}[19]$.
Here, $\mathfrak{D}$ is a subset of $\mathbb{R}^{3}$. In 2012, Bai and Sun reviewed the singular problem

$$
D_{0^{+}}^{\alpha} u(t)+f\left(t, u(t), D_{0^{+}}^{\nu} u(t), D_{0^{+}}^{\mu} u(t)\right)=0,
$$

$u(0)=u^{\prime}(0)=u^{\prime \prime}(0)=u^{\prime \prime}(1)=0$, where $3<\alpha \leq 4,0<v \leq$ $1,1<\mu \leq 2$ and $f$ satisfies the local Caratheodory condition on $[0,1] \times \mathfrak{D}[8]$. Again, $\mathfrak{D}$ is a subset of $\mathbb{R}^{3}$. In this year, Agarwal et al. reviewed the singular problem

$$
{ }^{c} D_{0^{+}}^{\alpha} u(t)+f\left(t, u(t), u^{\prime}(t),{ }^{c} D_{0^{+}}^{\mu} u(t)\right)=0,
$$

$u^{\prime}(0)=0, u(1)=0$, where $1<\alpha<2,0<\mu<$ 1 and $f$ is a $L^{q}$-Caratheodory function on $[0,1] \times \mathfrak{B}[2]$. Here, $\mathfrak{B}=(0, \infty) \times(-\infty, 0) \times(-\infty, 0), q>1 /(\alpha-1)$ and ${ }^{c} D$ is the Caputo fractional derivative. Many researchers have established the existence and uniqueness of solutions for some systems of nonlinear fractional differential equations, but there are few works about coupled system of multiterm nonlinear fractional differential equations (see, for example, $[4,5,20,21])$. Also, there are a few papers discussing singular system of fractional differential equations (see, e.g., $[6,16])$.

Let $\|x\|_{1}=\int_{0}^{1}|x(t)| d t$ be the norm of $L^{1}[0,1],\|x\|=$ $\max \{|x(t)|: t \in[0,1]\}$ the norm of $C[0,1]$, and $\|u\|_{*}=$ $\max \left\{\|u\|,\left\|u^{\prime}\right\|,\left\|u^{\prime \prime}\right\|\right\}$ the norm of $X=C^{2}[0,1]$. As you know, $\left(X,\|\cdot\|_{*}\right)$ and $\left(X \times X,\|\cdot\|_{* *}\right)$ are Banach spaces, where $\|(u, v)\|_{* *}=\max \left\{\|u\|_{*},\|v\|_{*}\right\}$. Suppose that $A C[0,1]$ and $A C^{k}[0,1]$ are the spaces of absolutely continuous functions and functions having absolutely continuous $k$ th derivatives on $[0,1]$, respectively. In this paper, we suppose that the functions $f_{1}$ and $f_{2}$ in (1) satisfy the following conditions:

$\left(H_{1}\right) f_{1}, f_{2} \in \operatorname{Car}([0,1] \times \mathfrak{D})$, where $\mathfrak{D}=(0, \infty)^{m+5}$. Also, there exists a positive constant $m_{j}$ such that $f_{j}\left(t, x, y, z, w, v, u_{1}, u_{2}, \ldots, u_{m}\right) \geq m_{j}$ for almost all $t \in[0,1]$ and $\left(x, y, z, w, v, u_{1}, u_{2}, \ldots, u_{m}\right) \in \mathfrak{D}$;

$\left(H_{2}\right)$ there exist positive mappings $k_{1}, k_{2} \in L^{1}[0,1]$, $P_{1}, P_{2} \in C(\mathfrak{D})$, and $h_{1}, h_{2} \in C\left([0, \infty)^{m+5}\right)$ such that $P_{1}$ and $P_{2}$ are nonincreasing and $h_{1}$ and $h_{2}$ are nondecreasing in all their variables,

$$
\begin{gathered}
M_{1}=\frac{m_{1}}{\left(\alpha-\delta_{1}\right) \Gamma(\alpha+1)}, \quad M_{2}=\frac{m_{2}}{\left(\beta-\delta_{2}\right) \Gamma(\beta+1)}, \\
\lim _{x \rightarrow \infty} \frac{h_{j}(x, x, \ldots, x)}{x}=0, \quad(j=1,2), \\
\int_{0}^{1} P_{1}\left(M_{1} s^{\alpha-1}, M_{2} s^{\beta-1},\right. \\
M_{1} \int_{0}^{s} \gamma_{1}(s, \tau) \tau^{\alpha-1} d \tau, M_{2} \int_{0}^{s} \lambda_{1}(s, \tau) \tau^{\beta-1} d \tau, \\
\frac{\left(\delta_{1}-2\right) M_{1}}{\Gamma(\alpha-p)} s^{\alpha-p}, \frac{\left(\delta_{2}-2\right) M_{2}}{\Gamma\left(\beta-\mu_{1}\right)} s^{\beta-\mu_{1}},
\end{gathered}
$$




$$
\begin{gathered}
\left.\frac{\left(\delta_{2}-2\right) M_{2}}{\Gamma\left(\beta-\mu_{2}\right)} s^{\beta-\mu_{2}}, \ldots, \frac{\left(\delta_{2}-2\right) M_{2}}{\Gamma\left(\beta-\mu_{m}\right)} s^{\beta-\mu_{m}}\right) d s<\infty, \\
\int_{0}^{1} P_{2}\left(M_{1} s^{\alpha-1}, M_{2} s^{\beta-1},\right. \\
M_{1} \int_{0}^{s} \gamma_{2}(s, \tau) \tau^{\alpha-1} d \tau, M_{2} \int_{0}^{s} \lambda_{2}(s, \tau) \tau^{\beta-1} d \tau, \\
\frac{\left(\delta_{2}-2\right) M_{2}}{\Gamma(\beta-q)} s^{\beta-q}, \frac{\left(\delta_{1}-2\right) M_{1}}{\Gamma\left(\alpha-v_{1}\right)} s^{\alpha-v_{1}}, \\
\left.\quad \frac{\left(\delta_{1}-2\right) M_{1}}{\Gamma\left(\alpha-v_{2}\right)} s^{\alpha-v_{2}}, \ldots, \frac{\left(\delta_{1}-2\right) M_{1}}{\Gamma\left(\alpha-v_{m}\right)} s^{\alpha-v_{m}}\right) d s<\infty, \\
f_{j}\left(t, x, y, z, w, v, u_{1}, u_{2}, \ldots, u_{m}\right) \\
\leq P_{j}\left(x, y, z, w, v, u_{1}, u_{2}, \ldots, u_{m}\right) \\
\quad+k h_{j}(t) h\left(x, y, z, w, v, u_{1}, u_{2}, \ldots, u_{m}\right)
\end{gathered}
$$

for almost all $t \in[0,1]$ and $\left(x, y, z, w, v, u_{1}, u_{2}, \ldots, u_{m}\right) \in \mathfrak{D}$. Since we suppose that the problem (1) is singular, we use regularization and sequential techniques for the existence of positive solutions of the problem. In this way, for each natural number $n$ define the function $f_{n}^{j}(j=1,2)$ by

$$
\begin{array}{r}
f_{n}^{j}\left(t, x, y, z, w, v, u_{1}, u_{2}, \ldots, u_{m}\right) \\
=f_{j}\left(t, \chi_{n}(x), \chi_{n}(y), \chi_{n}(z), \chi_{n}(w),\right. \\
\left.\quad \chi_{n}(v), \chi_{n}\left(u_{1}\right), \chi_{n}\left(u_{2}\right), \ldots, \chi_{n}\left(u_{m}\right)\right)
\end{array}
$$

for almost all $t \in[0,1]$ and $\left(x, y, z, w, v, u_{1}, u_{2}, \ldots, u_{m}\right) \in$ $\mathbb{R}^{m+5}$, where

$$
\chi_{n}(x)= \begin{cases}x & \text { if } x \geq \frac{1}{n} \\ \frac{1}{n} & \text { if } x<\frac{1}{n}\end{cases}
$$

Then condition $\left(H_{1}\right)$ implies that $f_{n}^{j} \in \operatorname{Car}\left([0,1] \times \mathbb{R}^{m+5}\right)$ and $f_{n}^{j}\left(t, x, y, z, w, v, u_{1}, u_{2}, \ldots, u_{m}\right) \geq m_{j}$ for almost all $t \in[0,1]$ and $\left(x, y, z, w, v, u_{1}, u_{2}, \ldots, u_{m}\right) \in \mathbb{R}^{m+5},(j=1,2)$. Also, the condition $\left(\mathrm{H}_{2}\right)$ implies

$$
\begin{aligned}
f_{n}^{j}\left(t, x, y, z, w, v, u_{1}, u_{2}, \ldots, u_{m}\right) & \\
\leq & P_{j}\left(\frac{1}{n}, \frac{1}{n}, \ldots, \frac{1}{n}\right) \\
& +k_{j}(t) h_{j}(1+x, 1+y, 1+z, 1+w, \\
& \left.1+v, 1+u_{1}, 1+u_{2}, \ldots, 1+u_{m}\right)
\end{aligned}
$$

for almost all $t \in[0,1]$ and $\left(x, y, z, w, v, u_{1}, u_{2}, \ldots, u_{m}\right) \in$ $[0, \infty)^{m+5}$ and

$$
\begin{aligned}
& f_{n}^{j}\left(t, x, y, z, w, v, u_{1}, u_{2}, \ldots, u_{m}\right) \\
& \leq P_{j}\left(x, y, z, w, v, u_{1}, u_{2}, \ldots, u_{m}\right) \\
& \quad+k_{j}(t) h_{j}(1+x, 1+y, 1+z, 1+w, \\
& \left.\quad 1+v, 1+u_{1}, 1+u_{2}, \ldots, 1+u_{m}\right)
\end{aligned}
$$

for almost all $t \in[0,1]$ and $\left(x, y, z, w, v, u_{1}, u_{2}, \ldots, u_{m}\right) \in \mathfrak{D}$. For obtaining solutions of the system (1), (2), and (3), for each natural number $n$, we will obtain solutions of the system

$$
\begin{gathered}
D_{0^{+}}^{\alpha} u(t)+f_{n}^{1}\left(t, u(t), v(t),\left(\phi_{1} u\right)(t),\left(\psi_{1} v\right)(t),\right. \\
\left.D_{0^{+}}^{p} u(t), D_{0^{+}}^{\mu_{1}} v(t), D_{0^{+}}^{\mu_{2}} v(t), \ldots, D_{0^{+}}^{\mu_{m}} v(t)\right)=0, \\
D_{0^{+}}^{\beta} v(t)+f_{n}^{2}\left(t, u(t), v(t),\left(\phi_{2} u\right)(t),\left(\psi_{2} v\right)(t),\right. \\
\left.D_{0^{+}}^{q} v(t), D_{0^{+}}^{\nu_{1}} u(t), D_{0^{+}}^{\nu_{2}} u(t), \ldots, D_{0^{+}}^{\nu_{m}} u(t)\right)=0,
\end{gathered}
$$

via the boundary conditions (2) and (3) and by using solutions of this system, we will obtain solution of the system (1), (2), and (3). It has been proved that the fractional integral $I_{0^{+}}^{\alpha}$ maps $L^{1}[0,1]$ into $L^{1}[0,1]$ whenever $\alpha \in(0,1)$ and maps $L^{1}[0,1]$ into $A C^{[\alpha]-1}[0,1]$ whenever $\alpha \geq 1[19]$. Here, $[\alpha]$ means the integral part of $\alpha$ and $A C^{0}[0,1]=A C[0,1]$. Suppose that $\alpha>0$ and $\alpha$ is not a natural number. If $x \in$ $C(0,1]$ and $D_{0^{+}}^{\alpha} x \in L^{1}[0,1]$, then $x(t)=I_{0^{+}}^{\alpha} D_{0^{+}}^{\alpha} x(t)+$ $\sum_{k=1}^{n} c_{k} t^{\alpha-k}$ for all $t \in(0,1]$, where $n=[\alpha]+1$ and $c_{k} \in \mathbb{R}$ for $k=1,2, \ldots, n$ [7]. Suppose that $v \in(0,1), \mu \in(1,2)$, $x \in C^{2}[0,1]$, and $x(0)=x^{\prime}(0)=0$. Then, $D_{0^{+}}^{\mu} x \in C[0,1]$, $D_{0^{+}}^{v} x \in C[0,1], D_{0^{+}}^{\mu} x(t)=(1 / \Gamma(2-\mu)) \int_{0}^{t}(t-s)^{1-\mu} x^{\prime \prime}(s) d s$, and $D_{0^{+}}^{v} x(t)=(1 / \Gamma(2-v)) \int_{0}^{t}(t-s)^{1-v} x^{\prime \prime}(s) d s[2]$.

\section{Main Results}

Now, we are ready to state and prove our main results. One can find main idea of next result in [17].

Lemma 1. Let $n \geq 4$ and $n-1<\alpha<n$. For each $g \in L^{1}[0,1]$, $u(t)=\int_{0}^{1} G_{\alpha}(t, s) g(s) d s$ is the unique solution of the equation $D_{0^{+}}^{\alpha} u(t)+g(t)=0$ in $C^{2}[0,1]$ which satisfies the boundary condition (2), where

$$
\begin{aligned}
& G_{\alpha}(t, s) \\
& \quad= \begin{cases}\frac{t^{\alpha-1}(1-s)^{\alpha-\delta_{1}-1}-(t-s)^{\alpha-1}}{\Gamma(\alpha)} & 0 \leq s \leq t \leq 1, \\
\frac{t^{\alpha-1}(1-s)^{\alpha-\delta_{1}-1}}{\Gamma(\alpha)} & 0 \leq t \leq s \leq 1 .\end{cases}
\end{aligned}
$$


Proof. It is easy to see that the functions $u(t)=-I_{0^{+}}^{\alpha} g(t)+$ $c_{1} t^{\alpha-1}+c_{2} t^{\alpha-2}+\cdots+c_{n} t^{\alpha-n}$ are solutions of $D_{0^{+}}^{\alpha} u(t)+g(t)=0$ in $C(0,1]$ for all $c_{i} \in \mathbb{R}$. Since $[\alpha] \geq 3, I_{0^{+}}^{\alpha} g \in A C^{[\alpha]-1}[0,1]$ and so $u(t)=-I_{0^{+}}^{\alpha} g(t)+c_{1} t^{\alpha-1}$ are solutions of $D_{0^{+}}^{\alpha} u(t)+g(t)=0$ in $C^{2}[0,1]$, where $c_{1} \in \mathbb{R}$. The boundary condition (2) implies that $c_{2}=\cdots=c_{n-1}=0$ and

$$
\begin{aligned}
0=c_{1} & \frac{\Gamma(\alpha)}{\Gamma\left(\alpha-\delta_{1}\right)}(1)^{\alpha-\delta_{1}-1} \\
& -\frac{1}{\Gamma\left(\alpha-\delta_{1}\right)} \int_{0}^{1}(1-s)^{\alpha-\delta_{1}-1} g(s) d s
\end{aligned}
$$

Thus, $u(t)=\left(t^{\alpha-1} / \Gamma(\alpha)\right) \int_{0}^{1}(1-s)^{\alpha-\delta_{1}-1} g(s) d s-(1 / \Gamma(\alpha)) \int_{0}^{t}(t-$ $s)^{\alpha-1} g(s) d s=\int_{0}^{1} G_{\alpha}(t, s) g(s) d s$ is the unique solution of the problem in $C^{2}[0,1]$.

Note that the Green function $G_{\alpha}$ in Lemma 1 has some properties. For example, $G_{\alpha},(\partial / \partial t) G_{\alpha}$ and $\left(\partial^{2} / \partial t^{2}\right) G_{\alpha}$ are continuous functions on $[0,1] \times[0,1], G_{\alpha}(t, s)>0$ on $(0,1) \times(0,1), G_{\alpha}(t, s) \leq 1 / \Gamma(\alpha)$ for all $(t, s) \in[0,1] \times[0,1]$, $\int_{0}^{1} G_{\alpha}(t, s) d s \geq t^{\alpha-1} /\left(\alpha-\delta_{1}\right) \Gamma(\alpha+1)$ for all $t \in[0,1]$, $(\partial / \partial t) G_{\alpha}(t, s)>0$ on $(0,1) \times(0,1),(\partial / \partial t) G_{\alpha}(t, s) \leq 1 / \Gamma(\alpha-1)$ for all $(t, s) \in[0,1] \times[0,1], \int_{0}^{1}(\partial / \partial t) G_{\alpha}(t, s) d s \geq t^{\alpha-2} /(\alpha-$ $\left.\delta_{1}\right) \Gamma(\alpha)$ for all $t \in[0,1],\left(\partial^{2} / \partial t^{2}\right) G_{\alpha}(t, s)>0$ on $(0,1) \times(0,1)$, $\left(\partial^{2} / \partial t^{2}\right) G_{\alpha}(t, s) \leq 1 / \Gamma(\alpha-2)$ for all $(t, s) \in[0,1] \times[0,1]$, and $\int_{0}^{1}\left(\partial^{2} / \partial t^{2}\right) G_{\alpha}(t, s) d s \geq\left(\delta_{1}-2\right) t^{\alpha-3} /\left(\alpha-\delta_{1}\right) \Gamma(\alpha-1)$ for all $t \in[0,1]$. Let $X=C^{2}[0,1]$. Define the cone $P$ on $X \times X$ by

$$
\begin{aligned}
P= & \{(x, y) \in X \times X: \\
& x(0)=x^{\prime}(0)=y(0)=y^{\prime}(0)=0, \\
& x(t), x^{\prime}(t), x^{\prime \prime}(t), y(t), y^{\prime}(t), y^{\prime \prime}(t) \geq 0, \\
& \forall t \in[0,1]\} .
\end{aligned}
$$

It is easy to see that for each $(x, y) \in P, i=1,2, \ldots, m$ and $t \in[0,1]$ we have $D_{0^{+}}^{p} x, D_{0^{+}}^{\nu_{i}} x, D_{0^{+}}^{q} y, D_{0^{+}}^{\mu_{i}} y \in C[0,1]$ and $D_{0^{+}}^{p} x(t) \geq 0, D_{0^{+}}^{\nu_{i}} x(t) \geq 0, D_{0^{+}}^{q} y(t) \geq 0, D_{0^{+}}^{\mu_{i}} y(t) \geq 0$. Now for each natural number $n$, define the operator $T_{n}$ on $P$ by

$$
\begin{aligned}
T_{n}(x, y)(t) \\
=\left(\begin{array}{l}
T_{n}^{1}(x, y)(t) \\
T_{n}^{2}(x, y)(t)
\end{array}\right) \\
=\left(\int_{0}^{1} G_{\alpha}(t, s) f_{n}^{1}\right.
\end{aligned}
$$

$$
\begin{gathered}
\times\left(s, x(s), y(s),\left(\phi_{1} x\right)(s),\right. \\
\left(\psi_{1} y\right)(s), D_{0^{+}}^{p} x(s), D_{0^{+}}^{\mu_{1}} y(s), \\
\left.D_{0^{+}}^{\mu_{2}} y(s), \ldots, D_{0^{+}}^{\mu_{m}} y(s)\right) d s \\
\int_{0}^{1} G_{\beta}(t, s) f_{n}^{2} \\
\times\left(s, x(s), y(s),\left(\phi_{2} x\right)(s),\right. \\
\left(\psi_{2} y\right)(s), D_{0^{+}}^{q} y(s), D_{0^{+}}^{\nu_{1}} x(s), \\
\left.\left.D_{0^{+}}^{\nu_{2}} x(s), \ldots, D_{0^{+}}^{\nu_{m}} x(s)\right) d s\right) .
\end{gathered}
$$

Lemma 2. For each natural number $n, T_{n}$ is a completely continuous operator on $P$.

Proof. Let $n$ be a natural number, $(x, y) \in P$,

$$
\begin{aligned}
& \rho^{1}(t)=f_{n}^{1}\left(t, x(t), y(t),\left(\phi_{1} x\right)(t),\left(\psi_{1} y\right)(t)\right. \\
&\left.D_{0^{+}}^{p} x(t), D_{0^{+}}^{\mu_{1}} y(t), D_{0^{+}}^{\mu_{2}} y(t), \ldots, D_{0^{+}}^{\mu_{m}} y(t)\right)
\end{aligned}
$$

and $\rho^{2}(t)=f_{n}^{2}\left(t, x(t), y(t), \quad\left(\phi_{2} x\right)(t), \quad\left(\psi_{2} y\right)(t), D_{0^{+}}^{q} y(t)\right.$, $\left.D_{0^{+}}^{\nu_{1}} x(t), D_{0^{+}}^{\nu_{2}} x(t), \ldots, D_{0^{+}}^{\nu_{m}} x(t)\right)$. Then, $\rho^{1}, \rho^{2} \in L^{1}[0,1]$ and there exist positive constants $m_{1}$ and $m_{2}$ such that $\rho^{1}(t) \geq m_{1}$ and $\rho^{2}(t) \geq m_{2}$ for almost all $t \in[0,1]$. Since $G_{\alpha}, G_{\beta},(\partial / \partial t) G_{\alpha},(\partial / \partial t) G_{\beta},\left(\partial^{2} / \partial t^{2}\right) G_{\alpha}$, and $\left(\partial^{2} / \partial t^{2}\right) G_{\beta}$ are nonnegative and continuous functions on $[0,1] \times[0,1]$, $G_{\alpha}(0, s)=0$, and $G_{\beta}(0, s)=0$ for all $s \in[0,1]$, we get $T_{n}^{1}(x, y), T_{n}^{2}(x, y) \in C^{2}[0,1], T_{n}^{1}(x, y)(0)=T_{n}^{2}(x, y)(0)=$ $\left(T_{n}^{1}(x, y)\right)^{\prime}(0)=\left(T_{n}^{2}(x, y)\right)^{\prime}(0)=0, T_{n}^{1}(x, y) \geq 0, T_{n}^{2}(x, y) \geq$ $0,\left(T_{n}^{1}(x, y)\right)^{\prime} \geq 0,\left(T_{n}^{2}(x, y)\right)^{\prime} \geq 0,\left(T_{n}^{1}(x, y)\right)^{\prime \prime} \geq 0$, and $\left(T_{n}^{2}(x, y)\right)^{\prime \prime} \geq 0$ on $[0,1]$. Thus, $T_{n}$ maps $P$ into $P$. Suppose that $\left\{\left(x_{k}, y_{k}\right)\right\}_{k \geq 1}$ is a convergent sequence in $P$ and $\lim _{k \rightarrow \infty}\left(x_{k}, y_{k}\right)=(x, y)$. Then, $\lim _{k \rightarrow \infty} x_{k}^{(j)}(t)=x^{(j)}(t)$ and $\lim _{k \rightarrow \infty} y_{k}^{(j)}(t)=y^{(j)}(t)$ uniformly on $[0,1]$ for $j=0,1,2$. Since for $p, q \in(0,1), \mu_{i}, v_{i} \in(1,2)$ for all $i=1,2, \ldots, m$,

$$
\begin{aligned}
& \left|D_{0^{+}}^{p} x_{k}(t)-D_{0^{+}}^{p} x(t)\right| \\
& \leq \frac{\left\|x_{k}^{\prime \prime}-x^{\prime \prime}\right\|}{\Gamma(2-p)} \int_{0}^{t}(t-s)^{1-p} d s \leq \frac{\left\|x_{k}^{\prime \prime}-x^{\prime \prime}\right\|}{\Gamma(3-p)}, \\
& \left|D_{0^{+}}^{q} y_{k}(t)-D_{0^{+}}^{q} y(t)\right| \\
& \quad \leq \frac{\left\|y_{k}^{\prime \prime}-y^{\prime \prime}\right\|}{\Gamma(2-q)} \int_{0}^{t}(t-s)^{1-q} d s \leq \frac{\left\|y_{k}^{\prime \prime}-y^{\prime \prime}\right\|}{\Gamma(3-q)}, \\
& \left|D_{0^{+}}^{v_{i}} x_{k}(t)-D_{0^{+}}^{v_{i}} x(t)\right| \\
& \quad \leq \frac{\left\|x_{k}^{\prime \prime}-x^{\prime \prime}\right\|}{\Gamma\left(2-v_{i}\right)} \int_{0}^{t}(t-s)^{1-v_{i}} d s \leq \frac{\left\|x_{k}^{\prime \prime}-x^{\prime \prime}\right\|}{\Gamma\left(3-v_{i}\right)},
\end{aligned}
$$




$$
\begin{aligned}
& \left|D_{0^{+}}^{\mu_{i}} y_{k}(t)-D_{0^{+}}^{\mu_{i}} y(t)\right| \\
& \quad \leq \frac{\left\|y_{k}^{\prime \prime}-y^{\prime \prime}\right\|}{\Gamma\left(2-\mu_{i}\right)} \int_{0}^{t}(t-s)^{1-\mu_{i}} d s \leq \frac{\left\|y_{k}^{\prime \prime}-y^{\prime \prime}\right\|}{\Gamma\left(3-\mu_{i}\right)}, \\
& \left|\left(\phi_{j} x_{k}\right)(t)-\left(\phi_{j} x\right)(t)\right| \\
& \quad \leq \sup _{t \in[0,1]}\left|\int_{0}^{t} \gamma_{j}(t, s) d s\right|\left\|x_{k}-x\right\|=\gamma_{0}^{j}\left\|x_{k}-x\right\|, \\
& \left|\left(\psi_{j} y_{k}\right)(t)-\left(\psi_{j} y\right)(t)\right| \\
& \quad \leq \sup _{t \in[0,1]}\left|\int_{0}^{t} \lambda_{j}(t, s) d s\right|\left\|y_{k}-y\right\|=\lambda_{0}^{j}\left\|y_{k}-y\right\|,
\end{aligned}
$$

for $j=1,2$ and $k \geq 1$, we have $\lim _{k \rightarrow \infty} D_{0^{+}}^{p} x_{k}(t)=$ $D_{0^{+}}^{p} x(t), \lim _{k \rightarrow \infty} D_{0^{+}}^{q} y_{k}(t)=D_{0^{+}}^{q} y(t), \lim _{k \rightarrow \infty} D_{0^{+}}^{v_{i}} x_{k}(t)=$ $D_{0^{+}}^{v_{i}} x(t), \lim _{k \rightarrow \infty} D_{0^{+}}^{\mu_{i}} y_{k}(t)=D_{0^{+}}^{\mu_{i}} y(t)$ for $i=1,2, \ldots, m$, and $\lim _{k \rightarrow \infty}\left(\phi_{j} x_{k}\right)(t)=\left(\phi_{j} x\right)(t), \lim _{k \rightarrow \infty}\left(\psi_{j} y_{k}\right)(t)=\left(\psi_{j} y\right)(t)$ uniformly on $[0,1]$ for $j=1,2$. Also, we have

$$
\begin{aligned}
\left\|D_{0^{+}}^{p} x_{k}\right\| & \leq \frac{\left\|x_{k}^{\prime \prime}\right\|}{\Gamma(2-p)} \int_{0}^{t}(t-s)^{1-p} d s \leq \frac{\left\|x_{k}^{\prime \prime}\right\|}{\Gamma(3-p)}, \\
\left\|D_{0^{+}}^{q} y_{k}\right\| & \leq \frac{\left\|y_{k}^{\prime \prime}\right\|}{\Gamma(2-q)} \int_{0}^{t}(t-s)^{1-q} d s \leq \frac{\left\|y_{k}^{\prime \prime}\right\|}{\Gamma(3-q)}, \\
\left\|D_{0^{+}}^{v_{i}} x_{k}\right\| & \leq \frac{\left\|x_{k}^{\prime \prime}\right\|}{\Gamma\left(2-v_{i}\right)} \int_{0}^{t}(t-s)^{1-v_{i}} d s \leq \frac{\left\|x_{k}^{\prime \prime}\right\|}{\Gamma\left(3-v_{i}\right)}, \\
\left\|D_{0^{+}}^{\mu_{i}} y_{k}\right\| & \leq \frac{\left\|y_{k}^{\prime \prime}\right\|}{\Gamma\left(2-\mu_{i}\right)} \int_{0}^{t}(t-s)^{1-\mu_{i}} d s \leq \frac{\left\|y_{k}^{\prime \prime}\right\|}{\Gamma\left(3-\mu_{i}\right)},
\end{aligned}
$$

and also $\left\|\phi_{j} x_{k}\right\| \leq \gamma_{0}^{j}\left\|x_{k}\right\|$ and $\left\|\psi_{j} y_{k}\right\| \leq \lambda_{0}^{j}\left\|y_{k}\right\|$ for $j=1,2$ and $k \geq 1$. Now, put

$$
\begin{gathered}
\rho_{k}^{1}(t)=f_{n}^{1}\left(t, x_{k}(t), y_{k}(t),\left(\phi_{1} x_{k}\right)(t),\right. \\
\left(\psi_{1} y_{k}\right)(t), D_{0^{+}}^{p} x_{k}(t), D_{0^{+}}^{\mu_{1}} y_{k}(t), \\
\left.D_{0^{+}}^{\mu_{2}} y_{k}(t), \ldots, D_{0^{+}}^{\mu_{m}} y_{k}(t)\right)
\end{gathered}
$$

and $\rho_{k}^{2}(t)=f_{n}^{2}\left(t, x_{k}(t), y_{k}(t),\left(\phi_{2} x_{k}\right)(t),\left(\psi_{2} y_{k}\right)(t), D_{0^{+}}^{q} y_{k}(t)\right.$, $\left.D_{0^{+}}^{\nu_{1}} x_{k}(t), D_{0^{+}}^{\nu_{2}} x_{k}(t), \ldots, D_{0^{+}}^{\nu_{m}} x_{k}(t)\right)$ for all $k \geq 1$. Then, $\lim _{k \rightarrow \infty} \rho_{k}^{j}(t)=\rho^{j}(t)$ for almost all $t \in[0,1]$ and $j=$ 1 ,2. Since $f_{n}^{j} \in \operatorname{Car}\left([0,1] \times \mathbb{R}^{m+5}\right)$ and $\left\{x_{k}\right\}_{k \geq 1},\left\{y_{k}\right\}_{k \geq 1}$ are bounded sequences in $C^{2}[0,1]$ and also by using the above inequalities, $\left\{D_{0^{+}}^{p} x_{k}\right\}_{k \geq 1},\left\{D_{0^{+}}^{q} y_{k}\right\}_{k \geq 1},\left\{D_{0^{+}}^{v_{i}} x_{k}\right\}_{k \geq 1},\left\{D_{0^{+}}^{\mu_{i}} y_{k}\right\}_{k \geq 1}$ for $i=1,2, \ldots, m$ and $\left\{\phi_{j} x_{k}\right\}_{k \geq 1},\left\{\psi_{j} x_{k}\right\}_{k \geq 1}$ for $j=1,2$ are bounded sequences in $C[0,1]$, there exist $g_{1}, g_{2} \in L^{1}[0,1]$ such that $m_{j} \leq \rho_{k}^{j}(t) \leq g_{j}(t)$ for almost all $t \in[0,1]$ and $k \geq 1$. Thus, by using the Lebesgue dominated convergence theorem and the following relations:

$$
\begin{aligned}
& \left|T_{n}^{1}\left(x_{k}, y_{k}\right)(t)-T_{n}^{1}(x, y)(t)\right| \\
& \quad \leq \frac{1}{\Gamma(\alpha)} \int_{0}^{1}\left|\rho_{k}^{1}(s)-\rho^{1}(s)\right| d s, \\
& \left|\left(T_{n}^{1}\left(x_{k}, y_{k}\right)\right)^{\prime}(t)-\left(T_{n}^{1}(x, y)\right)^{\prime}(t)\right| \\
& \quad \leq \frac{1}{\Gamma(\alpha-1)} \int_{0}^{1}\left|\rho_{k}^{1}(s)-\rho^{1}(s)\right| d s, \\
& \left|\left(T_{n}^{1}\left(x_{k}, y_{k}\right)\right)^{\prime \prime}(t)-\left(T_{n}^{1}(x, y)\right)^{\prime \prime}(t)\right| \\
& \quad \leq \frac{1}{\Gamma(\alpha-2)} \int_{0}^{1}\left|\rho_{k}^{1}(s)-\rho^{1}(s)\right| d s, \\
& \left|T_{n}^{2}\left(x_{k}, y_{k}\right)(t)-T_{n}^{2}(x, y)(t)\right| \\
& \quad \leq \frac{1}{\Gamma(\beta)} \int_{0}^{1}\left|\rho_{k}^{2}(s)-\rho^{2}(s)\right| d s, \\
& \left|\left(T_{n}^{2}\left(x_{k}, y_{k}\right)\right)^{\prime}(t)-\left(T_{n}^{2}(x, y)\right)^{\prime}(t)\right| \\
& \quad \leq \frac{1}{\Gamma(\beta-1)} \int_{0}^{1}\left|\rho_{k}^{2}(s)-\rho^{2}(s)\right| d s, \\
& \quad \leq \frac{1}{\Gamma(\beta-2)} \int_{0}^{1}\left|\rho_{k}^{2}(s)-\rho^{2}(s)\right| d s,
\end{aligned}
$$

we get $\lim _{k \rightarrow \infty}\left(T_{n}^{1}\left(x_{k}, y_{k}\right)\right)^{(j)}(t)=\left(T_{n}^{1}(x, y)\right)^{(j)}(t)$ and $\lim _{k \rightarrow \infty}\left(T_{n}^{2}\left(x_{k}, y_{k}\right)\right)^{(j)}(t)=\left(T_{n}^{2}(x, y)\right)^{(j)}(t)$ uniformly on $[0,1]$ for $j=0,1,2$. This implies that $T_{n}^{1}$ and $T_{n}^{2}$ are continuous operators and so $T_{n}$ is a continuous operator. Let $\left\{\left(x_{k}, y_{k}\right)\right\}_{k>1}$ be a bounded sequence in $P$. We show that the sequence $\left\{T_{n}\left(x_{k}, y_{k}\right)\right\}_{k \geq 1}$ is relatively compact in $X \times X$. By using the Arzela-Ascoli theorem, it is sufficient to prove that $\left\{T_{n}\left(x_{k}, y_{k}\right)\right\}_{k \geq 1}$ is bounded in $X \times X$ and $\left\{\left(T_{n}\left(x_{k}, y_{k}\right)\right)^{\prime \prime}\right\}$ is equicontinuous on $[0,1]$. Choose a positive number $S$ such that $\left\|x_{k}\right\| \leq S,\left\|y_{k}\right\| \leq S,\left\|x_{k}^{\prime}\right\| \leq S,\left\|y_{k}^{\prime}\right\| \leq S,\left\|x_{k}^{\prime \prime}\right\| \leq S$, $\left\|y_{k}^{\prime \prime}\right\| \leq S$ for all $k \geq 1$. By using the above inequalities, one can see that $\left\|D_{0^{+}}^{p} x_{k}\right\| \leq S / \Gamma(3-p),\left\|D_{0^{+}}^{q} y_{k}\right\| \leq S / \Gamma(3-q)$, $\left\|D_{0^{+}}^{v_{i}} x_{k}\right\| \leq S / \Gamma\left(3-v_{i}\right)$, and $\left\|D_{0^{+}}^{\mu_{i}} y_{k}\right\| \leq S / \Gamma\left(3-\mu_{i}\right)$ for $i=1,2, \ldots, m$ and $\left\|\phi_{j} x_{k}\right\| \leq \gamma_{0}^{j} S$ and $\left\|\psi_{j} x_{k}\right\| \leq \lambda_{0}^{j} S$ for $j=1,2$. Since

$$
\begin{aligned}
0 & \leq T_{n}^{1}\left(x_{k}, y_{k}\right)(t)=\int_{0}^{1} G_{\alpha}(t, s) \rho_{k}^{1}(s) d s \\
& \leq \frac{1}{\Gamma(\alpha)} \int_{0}^{1} g_{1}(s) d s=\frac{\left\|g_{1}\right\|_{1}}{\Gamma(\alpha)},
\end{aligned}
$$




$$
\begin{aligned}
0 & \leq\left(T_{n}^{1}\left(x_{k}, y_{k}\right)\right)^{\prime}(t)=\int_{0}^{1} \frac{\partial}{\partial t} G_{\alpha}(t, s) \rho_{k}^{1}(s) d s \\
& \leq \frac{1}{\Gamma(\alpha-1)} \int_{0}^{1} g_{1}(s) d s=\frac{\left\|g_{1}\right\|_{1}}{\Gamma(\alpha-1)}, \\
0 & \leq\left(T_{n}^{1}\left(x_{k}, y_{k}\right)\right)^{\prime \prime}(t)=\int_{0}^{1} \frac{\partial^{2}}{\partial t^{2}} G_{\alpha}(t, s) \rho_{k}^{1}(s) d s \\
& \leq \frac{1}{\Gamma(\alpha-2)} \int_{0}^{1} g_{1}(s) d s=\frac{\left\|g_{1}\right\|_{1}}{\Gamma(\alpha-2)},
\end{aligned}
$$

$\left\|T_{n}^{1}\left(x_{k}, y_{k}\right)\right\|_{*} \leq\left\|g_{1}\right\|_{1} / \Gamma(\alpha-2)$. Similarly, we can verify that $\left\|T_{n}^{2}\left(x_{k}, y_{k}\right)\right\|_{*} \leq\left\|g_{2}\right\|_{1} / \Gamma(\beta-2)$. Thus,

$$
\left\|T_{n}\left(x_{k}, y_{k}\right)\right\|_{* *} \leq \max \left\{\frac{\left\|g_{1}\right\|_{1}}{\Gamma(\alpha-2)}, \frac{\left\|g_{2}\right\|_{1}}{\Gamma(\beta-2)}\right\}
$$

for all $k$. Hence, $\left\{T_{n}\left(x_{k}, y_{k}\right)\right\}_{k \geq 1}$ is bounded in $X \times X$. Also, for $0 \leq t_{1}<t_{2} \leq 1$ we have

$$
\begin{aligned}
\left|\left(T_{n}^{1}\left(x_{k}, y_{k}\right)\right)^{\prime \prime}\left(t_{2}\right)-\left(T_{n}^{1}\left(x_{k}, y_{k}\right)\right)^{\prime \prime}\left(t_{1}\right)\right| \\
\leq \frac{t_{2}^{\alpha-3}-t_{1}^{\alpha-3}}{\Gamma(\alpha-2)} \int_{0}^{1}(1-s)^{\alpha-\delta_{1}-1} \rho_{k}^{1}(s) d s+\frac{1}{\Gamma(\alpha-2)} \\
\quad \times\left|\int_{0}^{t_{2}}\left(t_{2}-s\right)^{\alpha-3} \rho_{k}^{1}(s) d s-\int_{0}^{t_{1}}\left(t_{1}-s\right)^{\alpha-3} \rho_{k}^{1}(s) d s\right| \\
\leq \frac{\left\|\rho_{k}^{1}\right\|_{1}}{\Gamma(\alpha-2)}\left(t_{2}^{\alpha-3}-t_{1}^{\alpha-3}\right)+\frac{1}{\Gamma(\alpha-2)} \\
\quad \times\left[\int_{t_{1}}^{t_{2}}\left(t_{2}-s\right)^{\alpha-3} \rho_{k}^{1}(s) d s\right. \\
\left.\quad+\int_{0}^{t_{1}}\left(\left(t_{2}-s\right)^{\alpha-3}-\left(t_{1}-s\right)^{\alpha-3}\right) \rho_{k}^{1}(s) d s\right] \\
\leq \frac{\left\|g_{1}\right\|_{1}\left(t_{2}^{\alpha-3}-t_{1}^{\alpha-3}\right)+\frac{1}{\Gamma(\alpha-2)}}{\Gamma(\alpha-2)} \\
\quad \times\left[\left(t_{2}-t_{1}\right)^{\alpha-3}\left\|g_{1}\right\|_{1}^{t_{1}}\left(\left(t_{2}-s\right)^{\alpha-3}-\left(t_{1}-s\right)^{\alpha-3}\right) g_{1}(s) d s\right] .
\end{aligned}
$$

Similarly, we have

$$
\begin{aligned}
& \left|\left(T_{n}^{2}\left(x_{k}, y_{k}\right)\right)^{\prime \prime}\left(t_{2}\right)-\left(T_{n}^{2}\left(x_{k}, y_{k}\right)\right)^{\prime \prime}\left(t_{1}\right)\right| \\
& \leq \frac{\left\|g_{2}\right\|_{1}}{\Gamma(\beta-2)}\left(t_{2}^{\beta-3}-t_{1}^{\beta-3}\right)+\frac{1}{\Gamma(\beta-2)} \\
& \quad \times\left[\left(t_{2}-t_{1}\right)^{\beta-3}\left\|g_{2}\right\|_{1}\right. \\
& \left.\quad+\int_{0}^{t_{1}}\left(\left(t_{2}-s\right)^{\beta-3}-\left(t_{1}-s\right)^{\beta-3}\right) g_{2}(s) d s\right] .
\end{aligned}
$$

Let $\epsilon>0$ be given. Since the functions $t^{\alpha-3}$ and $t^{\beta-3}$ are uniformly continuous on $[0,1]$ and the functions $|t-s|^{\alpha-3}$ and $|t-s|^{\beta-3}$ are uniformly continuous on $[0,1] \times[0,1]$, there exists $\delta>0$ such that $0<t_{2}^{\alpha-3}-t_{1}^{\alpha-3}<\epsilon, 0<t_{2}^{\beta-3}-t_{1}^{\beta-3}<\epsilon$, $0<\left(t_{2}-s\right)^{\alpha-3}-\left(t_{1}-s\right)^{\alpha-3}<\epsilon$, and $0<\left(t_{2}-s\right)^{\beta-3}-\left(t_{1}-s\right)^{\beta-3}<\epsilon$ for all $0 \leq t_{1}<t_{2} \leq 1, t_{2}-t_{1}<\delta$ and $0 \leq s \leq t_{1}$. If $0 \leq t_{1}<t_{2} \leq 1$ and $t_{2}-t_{1}<\min \{\delta, \sqrt[\alpha-3]{\epsilon}, \sqrt[\beta-3]{\epsilon}\}$, then

$$
\left|\left(T_{n}^{1}\left(x_{k}, y_{k}\right)\right)^{\prime \prime}\left(t_{2}\right)-\left(T_{n}^{1}\left(x_{k}, y_{k}\right)\right)^{\prime \prime}\left(t_{1}\right)\right| \leq \frac{3 \epsilon}{\Gamma(\alpha-2)}\left\|g_{1}\right\|_{1}
$$

and $\left|\left(T_{n}^{2}\left(x_{k}, y_{k}\right)\right)^{\prime \prime}\left(t_{2}\right)-\left(T_{n}^{2}\left(x_{k}, y_{k}\right)\right)^{\prime \prime}\left(t_{1}\right)\right| \leq(3 \epsilon / \Gamma(\beta-$ 2)) $\left\|g_{2}\right\|_{1}$. Therefore, $\left\{\left(T_{n}\left(x_{k}, y_{k}\right)\right)^{\prime \prime}\right\}$ is equicontinuous on $[0,1]$ and so $T_{n}$ is a completely continuous operator on $P$.

We need the following result (see [10]).

Lemma 3. Let $Y$ be a Banach space, $P$ a cone in $Y$, and $\Omega_{1}$ and $\Omega_{2}$ bounded open balls in $Y$ centered at the origin with $\bar{\Omega}_{1} \subset \Omega_{2}$. Suppose that $T: P \cap\left(\bar{\Omega}_{2} \backslash \Omega_{1}\right) \rightarrow P$ is a completely continuous operator such that $\|T x\| \geq\|x\|$ for all $x \in P \cap \partial \Omega_{1}$ and $\|T x\| \leq\|x\|$ for all $x \in P \cap \partial \Omega_{2}$. Then $T$ has a fixed point in $P \cap\left(\bar{\Omega}_{2} \backslash \Omega_{1}\right)$.

Theorem 4. For each natural number $n$, the system (15), (2), and (3) has a solution $\left(u_{n}, v_{n}\right) \in P$ such that $u_{n} \geq M_{1} t^{\alpha-1}$ and $v_{n}(t) \geq M_{2} t^{\beta-1}$ for all $t \in[0,1]$.

Proof. Let $(x, y) \in P$ and $n \geq 1$ be given. Then, $T_{n}^{1}(x, y)(t) \geq m_{1} \int_{0}^{1} G_{\alpha}(t, s) d s \geq M_{1} t^{\alpha-1}$ and $T_{n}^{2}(x, y)(t) \geq$ $m_{2} \int_{0}^{1} G_{\beta}(t, s) d s \geq M_{2} t^{\beta-1}$ for all $t \in[0,1]$. Hence, $\left\|T_{n}^{1}(x, y)\right\| \geq M_{1}$ and $\left\|T_{n}^{2}(x, y)\right\| \geq M_{2}$ and so $\left\|T_{n}^{1}(x, y)\right\|_{*} \geq$ $M_{1}$ and $\left\|T_{n}^{2}(x, y)\right\|_{*} \geq M_{2}$. Thus, $\left\|T_{n}(x, y)\right\|_{* *} \geq$ $\max \left\{M_{1}, M_{2}\right\}=M$. Now, put

$$
\Omega_{1}=\left\{(x, y) \in X \times X:\|(x, y)\|_{* *}<M\right\} .
$$

Then, $\left\|T_{n}(x, y)\right\|_{* *} \geq\|(x, y)\|_{* *}$ for all $(x, y) \in P \cap \partial \Omega_{1}$. Let $W_{n}^{j}=P_{j}(1 / n, 1 / n, \ldots, 1 / n)$ for $j=1,2$. Then, we have

$$
\begin{aligned}
& 0 \leq\left(T_{n}^{1}(x, y)\right)^{\prime \prime}(t) \\
& \leq \frac{1}{\Gamma(\alpha-2)} \\
& \quad \times \int_{0}^{1}\left(W_{n}^{1}+k_{1}(s) h_{1}(1+x(s),\right. \\
& 1+y(s), 1+\left(\phi_{1} x\right)(s), \\
& 1+\left(\psi_{1} y\right)(s), 1+D_{0^{+}}^{p} x(s), \\
& 1+D_{0^{+}}^{\mu_{1}} y(s), 1+D_{0^{+}}^{\mu_{2}} y(s), \ldots, \\
& \left.\left.1+D_{0^{+}}^{\mu_{m}} y(s)\right)\right) d s
\end{aligned}
$$




$$
\begin{aligned}
& \leq \frac{1}{\Gamma(\alpha-2)} \\
& \times\left(W_{n}^{1}+h_{1}(1+\|x\|, 1+\|y\|,\right. \\
& 1+\left\|\phi_{1} x\right\|, 1+\left\|\psi_{1} y\right\|, \\
& 1+\left\|D_{0^{+}}^{p} x\right\|, 1+\left\|D_{0^{+}}^{\mu_{1}} y\right\|, \\
& 1+\left\|D_{0^{+}}^{\mu_{2}} y\right\|, \ldots, \\
& \left.\left.1+\left\|D_{0^{+}}^{\mu_{m}} y\right\|\right)\left\|k_{1}\right\|_{1}\right) \text {, } \\
& 0 \leq\left(T_{n}^{1}(x, y)\right)^{\prime}(t)=\int_{0}^{t}\left(T_{n}^{1}(x, y)\right)^{\prime \prime}(s) d s \\
& \leq \frac{1}{\Gamma(\alpha-2)} \\
& \times\left(W_{n}^{1}+h_{1}(1+\|x\|,\right. \\
& 1+\|y\|, 1+\left\|\phi_{1} x\right\|, 1+\left\|\psi_{1} y\right\| \\
& 1+\left\|D_{0^{+}}^{p} x\right\|, 1+\left\|D_{0^{+}}^{\mu_{1}} y\right\|, \\
& \left.\left.1+\left\|D_{0^{+}}^{\mu_{2}} y\right\|, \ldots, 1+\left\|D_{0^{+}}^{\mu_{m}} y\right\|\right)\left\|k_{1}\right\|_{1}\right) \\
& 0 \leq T_{n}^{1}(x, y)(t)=\int_{0}^{t}\left(T_{n}^{1}(x, y)\right)^{\prime}(s) d s \\
& \leq \frac{1}{\Gamma(\alpha-2)}\left(W_{n}^{1}+h_{1}(1+\|x\|, 1+\|y\|,\right. \\
& 1+\left\|\phi_{1} x\right\|, 1+\left\|\psi_{1} y\right\| \\
& 1+\left\|D_{0^{+}}^{p} x\right\|, 1+\left\|D_{0^{+}}^{\mu_{1}} y\right\|, \\
& \left.\left.1+\left\|D_{0^{+}}^{\mu_{2}} y\right\|, \ldots, 1+\left\|D_{0^{+}}^{\mu_{m}} y\right\|\right)\left\|k_{1}\right\|_{1}\right)
\end{aligned}
$$

for all $(x, y) \in P$ and $t \in[0,1]$. Hence,

$$
\begin{aligned}
& \left\|T_{n}^{1}(x, y)\right\|_{*} \\
& \leq \frac{1}{\Gamma(\alpha-2)}\left(W_{n}^{1}+h_{1}\left(1+\|x\|_{*},\right.\right. \\
& 1+\|y\|_{*}, 1+\gamma_{0}^{1}\|x\|_{*}, \\
& 1+\lambda_{0}^{1}\|y\|_{*}, 1+\frac{\|x\|_{*}}{\Gamma(3-p)}, \\
& 1+\frac{\|y\|_{*}}{\Gamma\left(3-\mu_{1}\right)}, 1+\frac{\|y\|_{*}}{\Gamma\left(3-\mu_{2}\right)}, \ldots, \\
& \left.\left.1+\frac{\|y\|_{*}}{\Gamma\left(3-\mu_{m}\right)}\right)\left\|k_{1}\right\|_{1}\right)
\end{aligned}
$$

$$
\begin{gathered}
\leq \frac{1}{\Gamma(\alpha-2)}\left(W_{n}^{1}+h_{1}\left(1+\|(x, y)\|_{* *},\right.\right. \\
1+\|(x, y)\|_{* *}, 1+\gamma_{0}^{1}\|(x, y)\|_{* *}, \\
1+\lambda_{0}^{1}\|(x, y)\|_{* *}, 1+\frac{\|(x, y)\|_{* *}}{\Gamma(3-p)} \\
1+\frac{\|(x, y)\|_{* *}}{\Gamma\left(3-\mu_{1}\right)} \\
1+\frac{\|(x, y)\|_{* *}}{\Gamma\left(3-\mu_{2}\right)}, \ldots, \\
\left.\left.1+\frac{\|(x, y)\|_{* *}}{\Gamma\left(3-\mu_{m}\right)}\right)\left\|k_{1}\right\|_{1}\right) .
\end{gathered}
$$

Similarly, we have

$$
\begin{aligned}
&\left\|T_{n}^{2}(x, y)\right\|_{*} \\
& \leq \frac{1}{\Gamma(\beta-2)}\left(W_{n}^{2}+h_{2}\left(1+\|(x, y)\|_{* *},\right.\right. \\
& 1+\|(x, y)\|_{* *}, 1+\gamma_{0}^{2}\|(x, y)\|_{* *}, \\
& 1+\lambda_{0}^{2}\|(x, y)\|_{* *}, 1+\frac{\|(x, y)\|_{* *}}{\Gamma(3-q)}, \\
& 1+\frac{\|(x, y)\|_{* *}}{\Gamma\left(3-v_{1}\right)}, 1+\frac{\|(x, y)\|_{* *}}{\Gamma\left(3-v_{2}\right)}, \ldots, \\
&\left.\left.1+\frac{\|(x, y)\|_{* *}}{\Gamma\left(3-v_{m}\right)}\right)\left\|k_{2}\right\|_{1}\right)
\end{aligned}
$$

for all $(x, y) \in P$. Since $\lim _{x \rightarrow \infty} h_{j}(x, x, \ldots, x) / x=0$ for $j=$ 1,2 , there exists $S>M>0$ such that

$$
\begin{aligned}
\frac{1}{\Gamma(\alpha-2)}\left(W_{n}^{1}+h_{1}(1+S,\right. & \\
& 1+S, 1+\gamma_{0}^{1} S, 1+\lambda_{0}^{1} S, \\
& 1+\frac{S}{\Gamma(3-p)}, 1+\frac{S}{\Gamma\left(3-\mu_{1}\right)}, \\
& 1+\frac{S}{\Gamma\left(3-\mu_{2}\right)}, \ldots, \\
& \left.\left.1+\frac{S}{\Gamma\left(3-\mu_{m}\right)}\right)\left\|k_{1}\right\|_{1}\right) \leq S,
\end{aligned}
$$




$$
\begin{aligned}
\frac{1}{\Gamma(\beta-2)}\left(W_{n}^{2}+h_{2}(1+S,\right. & \\
& 1+S, 1+\gamma_{0}^{2} S, 1+\lambda_{0}^{2} S \\
& 1+\frac{S}{\Gamma(3-q)}, 1+\frac{S}{\Gamma\left(3-\nu_{1}\right)}, \\
& 1+\frac{S}{\Gamma\left(3-v_{2}\right)}, \ldots, \\
& \left.\left.1+\frac{S}{\Gamma\left(3-v_{m}\right)}\right)\left\|k_{2}\right\|_{1}\right) \leq S .
\end{aligned}
$$

Put $\Omega_{2}=\left\{(x, y) \in X \times X\right.$ s.t $\left.\|(x, y)\|_{* *}<S\right\}$. Then, the above inequalities imply that $\left\|T_{n}(x, y)\right\|_{* *} \leq\|(x, y)\|_{* *}$ for all $(x, y) \in P \cap \partial \Omega_{2}$. Now by using Lemma 3 , we conclude that the operator $T_{n}$ has a fixed point $\left(u_{n}, v_{n}\right)$ in $P \cap\left(\bar{\Omega}_{2} \backslash \Omega_{1}\right)$. It is easy to see that $\left(u_{n}, v_{n}\right)$ is a desired solution of the system, that is, $u_{n} \geq M_{1} t^{\alpha-1}$ and $v_{n}(t) \geq M_{2} t^{\beta-1}$ for all $t \in[0,1]$.

We need the following lemma.

Lemma 5. The set of solutions $\left\{\left(u_{n}, v_{n}\right)\right\}_{n \geq 1}$ of the system (15), (2), and (3) is a relatively compact subset of $X \times X$.

Proof. It is easy to check that

$$
\begin{aligned}
& u_{n}(t) \\
& =\int_{0}^{1} G_{\alpha}(t, s) f_{n}^{1}\left(s, u_{n}(s), v_{n}(s),\right. \\
& \\
& \quad\left(\phi_{1} u_{n}\right)(s),\left(\psi_{1} v_{n}\right)(s), D_{0^{+}}^{p} u_{n}(s), \\
& D_{0^{+}}^{\mu_{1}} v_{n}(s), D_{0^{+}}^{\mu_{2}} v_{n}(s), \ldots, \\
& \left.D_{0^{+}}^{\mu_{m}} v_{n}(s)\right) d s,
\end{aligned}
$$

$v_{n}(t)$

$$
\begin{aligned}
=\int_{0}^{1} G_{\beta}(t, s) f_{n}^{2}\left(s, u_{n}(s),\right. & \\
& v_{n}(s),\left(\phi_{2} u_{n}\right)(s),\left(\psi_{2} v_{n}\right)(s), \\
& D_{0^{+}}^{q} v_{n}(s), D_{0^{+}}^{\nu_{1}} u_{n}(s), \\
& \left.D_{0^{+}}^{\nu_{2}} u_{n}(s), \ldots, D_{0^{+}}^{\gamma_{m}} u_{n}(s)\right) d s
\end{aligned}
$$

for $t \in[0,1]$ and $n \geq 1$, satisfy in Theorem 4. Also, $u_{n}^{\prime}(t) \geq m_{1} \int_{0}^{1}(\partial / \partial t) G_{\alpha}(t, s) d s \geq m_{1} t^{\alpha-2} / \Gamma(\alpha)\left(\alpha-\delta_{1}\right), u_{n}^{\prime \prime}(t) \geq$ $m_{1} \int_{0}^{1}\left(\partial^{2} / \partial t^{2}\right) G_{\alpha}(t, s) d s \geq m_{1}\left(\delta_{1}-2\right) t^{\alpha-3} / \Gamma(\alpha-1)\left(\alpha-\delta_{1}\right)$, $v_{n}^{\prime}(t) \geq m_{2} \int_{0}^{1}(\partial / \partial t) G_{\beta}(t, s) d s \geq m_{2} t^{\beta-2} / \Gamma(\beta)\left(\beta-\delta_{2}\right)$, and $v_{n}^{\prime \prime}(t) \geq m_{2} \int_{0}^{1}\left(\partial^{2} / \partial t^{2}\right) G_{\beta}(t, s) d s \geq m_{2}\left(\delta_{2}-2\right) t^{\beta-3} / \Gamma(\beta-1)(\beta-$ $\delta_{2}$ ) for $t \in[0,1]$ and $n \geq 1$. Moreover,

$$
\begin{aligned}
& D_{0^{+}}^{p} u_{n}(t) \\
& =\frac{1}{\Gamma(2-p)} \int_{0}^{t}(t-s)^{1-p} u_{n}^{\prime \prime}(s) d s \\
& \geq \frac{m_{1}\left(\delta_{1}-2\right)}{\Gamma(2-p) \Gamma(\alpha-1)\left(\alpha-\delta_{1}\right)} \\
& \times \int_{0}^{t}(t-s)^{1-p} s^{\alpha-3} d s, \\
& D_{0^{+}}^{q} v_{n}(t) \\
& =\frac{1}{\Gamma(2-q)} \int_{0}^{t}(t-s)^{1-q} v_{n}^{\prime \prime}(s) d s \\
& \geq \frac{m_{2}\left(\delta_{2}-2\right)}{\Gamma(2-q) \Gamma(\beta-1)\left(\beta-\delta_{2}\right)} \\
& \times \int_{0}^{t}(t-s)^{1-q} s^{\beta-3} d s, \\
& D_{0^{+}}^{v_{i}} u_{n}(t) \\
& =\frac{1}{\Gamma\left(2-v_{i}\right)} \int_{0}^{t}(t-s)^{1-v_{i}} u_{n}^{\prime \prime}(s) d s \\
& \geq \frac{m_{1}\left(\delta_{1}-2\right)}{\Gamma\left(2-v_{i}\right) \Gamma(\alpha-1)\left(\alpha-\delta_{1}\right)} \\
& \times \int_{0}^{t}(t-s)^{1-v_{i}} s^{\alpha-3} d s, \\
& D_{0^{+}}^{\mu_{i}} v_{n}(t) \\
& =\frac{1}{\Gamma\left(2-\mu_{i}\right)} \int_{0}^{t}(t-s)^{1-\mu_{i}} v_{n}^{\prime \prime}(s) d s \\
& \geq \frac{m_{2}\left(\delta_{2}-2\right)}{\Gamma\left(2-\mu_{i}\right) \Gamma(\beta-1)\left(\beta-\delta_{2}\right)} \\
& \times \int_{0}^{t}(t-s)^{1-\mu_{i}} s^{\beta-3} d s
\end{aligned}
$$

for $i=1,2, \ldots, m$. Since

$$
\begin{gathered}
\int_{0}^{t}(t-s)^{1-p} s^{\alpha-3} d s=t^{\alpha-p-1} \\
\quad \times \int_{0}^{1}(1-\xi)^{1-p} \xi^{\alpha-3} d \xi \\
=\frac{\Gamma(2-p) \Gamma(\alpha-2)}{\Gamma(\alpha-p)} t^{\alpha-p-1} \\
\geq \frac{\Gamma(2-p) \Gamma(\alpha-2)}{\Gamma(\alpha-p)} t^{\alpha-p},
\end{gathered}
$$


Abstract and Applied Analysis

9

$$
\begin{aligned}
& \int_{0}^{t}(t-s)^{1-q} s^{\beta-3} d s \geq \frac{\Gamma(2-q) \Gamma(\beta-2)}{\Gamma(\beta-q)} t^{\beta-q}, \\
& \int_{0}^{t}(t-s)^{1-v_{i}} s^{\alpha-3} d s \geq \frac{\Gamma\left(2-v_{i}\right) \Gamma(\alpha-2)}{\Gamma\left(\alpha-v_{i}\right)} t^{\alpha-v_{i}}, \\
& \int_{0}^{t}(t-s)^{1-\mu_{i}} s^{\beta-3} d s \geq \frac{\Gamma\left(2-\mu_{i}\right) \Gamma(\beta-2)}{\Gamma\left(\beta-\mu_{i}\right)} t^{\beta-\mu_{i}}
\end{aligned}
$$

for $i=1,2, \ldots, m$, we get $D_{0^{+}}^{p} u_{n}(t) \geq\left(m_{1}\left(\delta_{1}-2\right) /(\alpha-2)(\alpha-\right.$ $\left.\left.\delta_{1}\right) \Gamma(\alpha-p)\right) t^{\alpha-p}, D_{0^{+}}^{q} v_{n}(t) \geq\left(m_{2}\left(\delta_{2}-2\right) /(\beta-2)\left(\beta-\delta_{2}\right) \Gamma(\beta-\right.$ $q)) t^{\beta-q}, D_{0^{+}}^{v_{i}} u_{n}(t) \geq\left(m_{1}\left(\delta_{1}-2\right) /(\alpha-2)\left(\alpha-\delta_{1}\right) \Gamma\left(\alpha-v_{i}\right)\right) t^{\alpha-v_{i}}$, and $D_{0^{+}}^{\mu_{i}} v_{n}(t) \geq\left(m_{2}\left(\delta_{2}-2\right) /(\beta-2)\left(\beta-\delta_{2}\right) \Gamma\left(\beta-\mu_{i}\right)\right) t^{\beta-\mu_{i}}$ for all $t \in[0,1]$ and $n \geq 1$. Since

$$
m_{1} \cdot \min \left\{\frac{1}{\left(\alpha-\delta_{1}\right) \Gamma(\alpha+1)}, \frac{1}{(\alpha-2)\left(\alpha-\delta_{1}\right)}\right\}=M_{1}
$$

and $m_{2} \cdot \min \left\{1 /\left(\beta-\delta_{2}\right) \Gamma(\beta+1), 1 /(\beta-2)\left(\beta-\delta_{2}\right)\right\}=M_{2}$, for each $t \in[0,1]$ and $n \geq 1$, we get $u_{n}(t) \geq M_{1} t^{\alpha-1}, v_{n}(t) \geq$ $M_{2} t^{\beta-1}, D_{0^{+}}^{p} u_{n}(t) \geq\left(\left(\delta_{1}-2\right) M_{1} / \Gamma(\alpha-p)\right) t^{\alpha-p}, D_{0^{+}}^{q} v_{n}(t) \geq$ $\left(\left(\delta_{2}-2\right) M_{2} / \Gamma(\beta-q)\right) t^{\beta-q}, D_{0^{+}}^{\nu_{i}} u_{n}(t) \geq\left(\left(\delta_{1}-2\right) M_{1} / \Gamma(\alpha-\right.$ $\left.\left.v_{i}\right)\right) t^{\alpha-v_{i}}$, and $D_{0^{+}}^{\mu_{i}} v_{n}(t) \geq\left(\left(\delta_{2}-2\right) M_{2} / \Gamma\left(\beta-\mu_{i}\right)\right) t^{\beta-\mu_{i}}$ for $i=1,2, \ldots, m$ and $\left(\phi_{j} u_{n}\right)(t) \geq M_{1} \int_{0}^{t} \gamma_{j}(t, s) s^{\alpha-1} d s$ and $\left(\psi_{j} v_{n}\right)(t) \geq M_{2} \int_{0}^{t} \lambda_{j}(t, s) s^{\beta-1} d s$ for $j=1,2$. Thus,

$$
\begin{gathered}
P_{1}\left(u_{n}(t), v_{n}(t),\left(\phi_{1} u_{n}\right)(t),\left(\psi_{1} v_{n}\right)(t),\right. \\
\left.D_{0^{+}}^{p} u_{n}(t), D_{0^{+}}^{\mu_{1}} v_{n}(t), D_{0^{+}}^{\mu_{2}} v_{n}(t), \ldots, D_{0^{+}}^{\mu_{m}} v_{n}(t)\right) \\
\leq P_{1}\left(M_{1} t^{\alpha-1}, M_{2} t^{\beta-1}, M_{1} \int_{0}^{t} \gamma_{1}(t, s) s^{\alpha-1} d s,\right. \\
M_{2} \int_{0}^{t} \lambda_{1}(t, s) s^{\beta-1} d s, \frac{\left(\delta_{1}-2\right) M_{1}}{\Gamma(\alpha-p)} t^{\alpha-p}, \\
\frac{\left(\delta_{2}-2\right) M_{2}}{\Gamma\left(\beta-\mu_{1}\right)} t^{\beta-\mu_{1}}, \frac{\left(\delta_{2}-2\right) M_{2}}{\Gamma\left(\beta-\mu_{2}\right)} t^{\beta-\mu_{2}}, \ldots, \\
\left.\frac{\left(\delta_{2}-2\right) M_{2}}{\Gamma\left(\beta-\mu_{m}\right)} t^{\beta-\mu_{m}}\right)
\end{gathered}
$$

$$
\begin{gathered}
P_{2}\left(u_{n}(t), v_{n}(t),\left(\phi_{2} u_{n}\right)(t),\left(\psi_{2} v_{n}\right)(t), D_{0^{+}}^{q} v_{n}(t),\right. \\
\left.D_{0^{+}}^{\nu_{1}} u_{n}(t), D_{0^{+}}^{\nu_{2}} u_{n}(t), \ldots, D_{0^{+}}^{v_{m}} u_{n}(t)\right) \\
\leq P_{2}\left(M_{1} t^{\alpha-1}, M_{2} t^{\beta-1}, M_{1} \int_{0}^{t} \gamma_{2}(t, s) s^{\alpha-1} d s\right. \\
M_{2} \int_{0}^{t} \lambda_{2}(t, s) s^{\beta-1} d s, \frac{\left(\delta_{2}-2\right) M_{2}}{\Gamma(\beta-q)} t^{\beta-q}, \\
\frac{\left(\delta_{1}-2\right) M_{1}}{\Gamma\left(\alpha-v_{1}\right)} t^{\alpha-v_{1}}, \frac{\left(\delta_{1}-2\right) M_{1}}{\Gamma\left(\alpha-v_{2}\right)} t^{\alpha-v_{2}}, \ldots, \\
\left.\frac{\left(\delta_{1}-2\right) M_{1}}{\Gamma\left(\alpha-v_{m}\right)} t^{\alpha-v_{m}}\right)
\end{gathered}
$$

$$
\begin{aligned}
& 0 \leq u_{n}^{\prime \prime}(t) \\
& =\int_{0}^{1} \frac{\partial^{2}}{\partial t^{2}} G_{\alpha}(t, s) f_{n}^{1}\left(s, u_{n}(s), v_{n}(s)\right. \text {, } \\
& \left(\phi_{1} u_{n}\right)(s),\left(\psi_{1} v_{n}\right)(s), \\
& D_{0^{+}}^{p} u_{n}(s), D_{0^{+}}^{\mu_{1}} v_{n}(s), \\
& \left.D_{0^{+}}^{\mu_{2}} v_{n}(s), \ldots, D_{0^{+}}^{\mu_{m}} v_{n}(s)\right) d s \\
& \leq \frac{1}{\Gamma(\alpha-2)}\left[\int _ { 0 } ^ { 1 } P _ { 1 } \left(M_{1} s^{\alpha-1}, M_{2} s^{\beta-1},\right.\right. \\
& M_{1} \int_{0}^{s} \gamma_{1}(s, \tau) \tau^{\alpha-1} d \tau, \\
& M_{2} \int_{0}^{s} \lambda_{1}(s, \tau) \tau^{\beta-1} d \tau, \\
& \frac{\left(\delta_{1}-2\right) M_{1}}{\Gamma(\alpha-p)} s^{\alpha-p}, \\
& \frac{\left(\delta_{2}-2\right) M_{2}}{\Gamma\left(\beta-\mu_{1}\right)} s^{\beta-\mu_{1}}, \\
& \frac{\left(\delta_{2}-2\right) M_{2}}{\Gamma\left(\beta-\mu_{2}\right)} s^{\beta-\mu_{2}}, \ldots, \\
& \left.\frac{\left(\delta_{2}-2\right) M_{2}}{\Gamma\left(\beta-\mu_{m}\right)} s^{\beta-\mu_{m}}\right) d s \\
& +h_{1}\left(1+\left\|u_{n}\right\|_{*}, 1+\left\|v_{n}\right\|_{*},\right. \\
& 1+\gamma_{0}^{1}\left\|u_{n}\right\|_{*}, 1+\lambda_{0}^{1}\left\|v_{n}\right\|_{*},
\end{aligned}
$$




$$
\begin{aligned}
& 1+\frac{\left\|u_{n}\right\|_{*}}{\Gamma(3-p)}, 1+\frac{\left\|v_{n}\right\|_{*}}{\Gamma\left(3-\mu_{1}\right)}, \\
& 1+\frac{\left\|u_{n}\right\|_{*}}{\Gamma(3-p)}, 1+\frac{\left\|v_{n}\right\|_{*}}{\Gamma\left(3-\mu_{1}\right)}, \\
& \left.1+\frac{\left\|v_{n}\right\|_{*}}{\Gamma\left(3-\mu_{2}\right)}, \ldots, 1+\frac{\left\|v_{n}\right\|_{*}}{\Gamma\left(3-\mu_{m}\right)}\right) \\
& 1+\frac{\left\|v_{n}\right\|_{*}}{\Gamma\left(3-\mu_{2}\right)}, \ldots, \\
& \left.\times \int_{0}^{1} k_{1}(s) d s\right] \\
& \left.\left.1+\frac{\left\|v_{n}\right\|_{*}}{\Gamma\left(3-\mu_{m}\right)}\right)\left\|k_{1}\right\|_{1}\right) \\
& =\frac{1}{\Gamma(\alpha-2)} \\
& \times\left(\Lambda_{1}+h_{1}\left(1+\left\|u_{n}\right\|_{*}, 1+\left\|v_{n}\right\|_{*},\right.\right. \\
& 1+\gamma_{0}^{1}\left\|u_{n}\right\|_{*}, 1+\lambda_{0}^{1}\left\|v_{n}\right\|_{*}, \\
& 1+\frac{\left\|u_{n}\right\|_{*}}{\Gamma(3-p)}, 1+\frac{\left\|v_{n}\right\|_{*}}{\Gamma\left(3-\mu_{1}\right)}, \\
& 1+\frac{\left\|v_{n}\right\|_{*}}{\Gamma\left(3-\mu_{2}\right)}, \ldots, \\
& \left.\left.1+\frac{\left\|v_{n}\right\|_{*}}{\Gamma\left(3-\mu_{m}\right)}\right)\left\|k_{1}\right\|_{1}\right) \text {, } \\
& 0 \leq u_{n}^{\prime}(t)=\int_{0}^{t} u_{n}^{\prime \prime}(s) d s \\
& \leq \frac{1}{\Gamma(\alpha-2)} \\
& \times\left(\Lambda_{1}+h_{1}\left(1+\left\|u_{n}\right\|_{*},\right.\right. \\
& 1+\left\|v_{n}\right\|_{*}, 1+\gamma_{0}^{1}\left\|u_{n}\right\|_{*}, \\
& 1+\lambda_{0}^{1}\left\|v_{n}\right\|_{*}, \\
& 1+\frac{\left\|u_{n}\right\|_{*}}{\Gamma(3-p)}, 1+\frac{\left\|v_{n}\right\|_{*}}{\Gamma\left(3-\mu_{1}\right)}, \\
& 1+\frac{\left\|v_{n}\right\|_{*}}{\Gamma\left(3-\mu_{2}\right)}, \ldots, \\
& \left.\left.1+\frac{\left\|v_{n}\right\|_{*}}{\Gamma\left(3-\mu_{m}\right)}\right)\left\|k_{1}\right\|_{1}\right) \\
& \Lambda_{1}=\int_{0}^{1} P_{1}\left(M_{1} s^{\alpha-1}, M_{2} s^{\beta-1},\right. \\
& M_{1} \int_{0}^{s} \gamma_{1}(s, \tau) \tau^{\alpha-1} d \tau, \\
& M_{2} \int_{0}^{s} \lambda_{1}(s, \tau) \tau^{\beta-1} d \tau, \\
& \frac{\left(\delta_{1}-2\right) M_{1}}{\Gamma(\alpha-p)} s^{\alpha-p}, \frac{\left(\delta_{2}-2\right) M_{2}}{\Gamma\left(\beta-\mu_{1}\right)} s^{\beta-\mu_{1}}, \\
& \frac{\left(\delta_{2}-2\right) M_{2}}{\Gamma\left(\beta-\mu_{2}\right)} s^{\beta-\mu_{2}}, \ldots, \\
& \left.\frac{\left(\delta_{2}-2\right) M_{2}}{\Gamma\left(\beta-\mu_{m}\right)} s^{\beta-\mu_{m}}\right) d s . \\
& \left\|u_{n}\right\|_{*} \leq \frac{1}{\Gamma(\alpha-2)}\left(\Lambda_{1}+h_{1}\left(1+\left\|u_{n}\right\|_{*},\right.\right. \\
& 1+\left\|v_{n}\right\|_{*}, 1+\gamma_{0}^{1}\left\|u_{n}\right\|_{*}, \\
& 0 \leq u_{n}(t)=\int_{0}^{t} u_{n}^{\prime}(s) d s \\
& \leq \frac{1}{\Gamma(\alpha-2)} \\
& \times\left(\Lambda_{1}+h_{1}\left(1+\left\|u_{n}\right\|_{*},\right.\right. \\
& 1+\left\|v_{n}\right\|_{*}, 1+\gamma_{0}^{1}\left\|u_{n}\right\|_{*}, \\
& 1+\lambda_{0}^{1}\left\|v_{n}\right\|_{*}, \\
& 1+\lambda_{0}^{1}\left\|v_{n}\right\|_{*}, \\
& 1+\frac{\left\|u_{n}\right\|_{*}}{\Gamma(3-p)}, 1+\frac{\left\|v_{n}\right\|_{*}}{\Gamma\left(3-\mu_{1}\right)}, \\
& 1+\frac{\left\|v_{n}\right\|_{*}}{\Gamma\left(3-\mu_{2}\right)}, \ldots, \\
& \left.\left.1+\frac{\left\|v_{n}\right\|_{*}}{\Gamma\left(3-\mu_{m}\right)}\right)\left\|k_{1}\right\|_{1}\right)
\end{aligned}
$$




$$
\begin{aligned}
\leq \frac{1}{\Gamma(\alpha-2)}\left(\Lambda_{1}+h_{1}\left(1+\left\|\left(u_{n}, v_{n}\right)\right\|_{* *},\right.\right. & \\
1 & +\left\|\left(u_{n}, v_{n}\right)\right\|_{* *}, 1+\gamma_{0}^{1}\left\|\left(u_{n}, v_{n}\right)\right\|_{* *}, \\
1 & \lambda_{0}^{1}\left\|\left(u_{n}, v_{n}\right)\right\|_{* *}, \\
1 & +\frac{\left\|\left(u_{n}, v_{n}\right)\right\|_{* *}}{\Gamma(3-p)}, 1+\frac{\left\|\left(u_{n}, v_{n}\right)\right\|_{* *}}{\Gamma\left(3-\mu_{1}\right)}, \\
1 & \frac{\left\|\left(u_{n}, v_{n}\right)\right\|_{* *}}{\Gamma\left(3-\mu_{2}\right)}, \ldots, \\
1 & \left.\left.+\frac{\left\|\left(u_{n}, v_{n}\right)\right\|_{* *}}{\Gamma\left(3-\mu_{m}\right)}\right)\left\|k_{1}\right\|_{1}\right)=G_{n}^{1}
\end{aligned}
$$

for all $n \geq 1$. Similarly, we have

$$
\begin{aligned}
&\left\|v_{n}\right\|_{*} \leq \frac{1}{\Gamma(\beta-2)} \\
& \times\left(\Lambda_{2}+h_{2}\left(1+\left\|\left(u_{n}, v_{n}\right)\right\|_{* *}, 1+\left\|\left(u_{n}, v_{n}\right)\right\|_{* *},\right.\right. \\
& 1+\gamma_{0}^{2}\left\|\left(u_{n}, v_{n}\right)\right\|_{* *}, \\
& 1+\lambda_{0}^{2}\left\|\left(u_{n}, v_{n}\right)\right\|_{* *}, \\
& 1+\frac{\left\|\left(u_{n}, v_{n}\right)\right\|_{* *}}{\Gamma(3-q)}, 1+\frac{\left\|\left(u_{n}, v_{n}\right)\right\|_{* *}}{\Gamma\left(3-v_{1}\right)}, \\
& 1+\frac{\left\|\left(u_{n}, v_{n}\right)\right\|_{* *}}{\Gamma\left(3-v_{2}\right)}, \ldots, \\
&\left.\left.1+\frac{\left\|\left(u_{n}, v_{n}\right)\right\|_{* *}}{\Gamma\left(3-v_{m}\right)}\right)\left\|k_{2}\right\|_{1}\right)=G_{n}^{2}
\end{aligned}
$$

for all $n \geq 1$, where

$$
\begin{gathered}
\Lambda_{2}=\int_{0}^{1} P_{2}\left(M_{1} s^{\alpha-1}, M_{2} s^{\beta-1},\right. \\
M_{1} \int_{0}^{s} \gamma_{2}(s, \tau) \tau^{\alpha-1} d \tau, \\
M_{2} \int_{0}^{s} \lambda_{2}(s, \tau) \tau^{\beta-1} d \tau, \\
\frac{\left(\delta_{2}-2\right) M_{2}}{\Gamma(\beta-q)} s^{\beta-q},
\end{gathered}
$$

$$
\begin{aligned}
& \frac{\left(\delta_{1}-2\right) M_{1}}{\Gamma\left(\alpha-v_{1}\right)} s^{\alpha-v_{1}}, \\
& \frac{\left(\delta_{1}-2\right) M_{1}}{\Gamma\left(\alpha-v_{2}\right)} s^{\alpha-v_{2}}, \ldots, \\
& \left.\frac{\left(\delta_{1}-2\right) M_{1}}{\Gamma\left(\alpha-v_{m}\right)} s^{\alpha-v_{m}}\right) d s .
\end{aligned}
$$

Thus, $\left\|\left(u_{n}, v_{n}\right)\right\|_{* *} \leq \max \left\{G_{n}^{1}, G_{n}^{2}\right\}$ for all $n \geq 1$. Since $\lim _{x \rightarrow \infty} h_{j}(x, x, \ldots, x) / x=0$ for $j=1,2$, there exists $L>0$ such that

$$
\frac{1}{\Gamma(\alpha-2)}\left(\Lambda_{1}+h_{1}\left(1+v, 1+v, 1+\gamma_{0}^{1} v\right.\right.
$$

$$
\begin{aligned}
& 1+\lambda_{0}^{1} v, 1+\frac{v}{\Gamma(3-p)}, \\
& 1+\frac{v}{\Gamma\left(3-\mu_{1}\right)}, \\
& 1+\frac{v}{\Gamma\left(3-\mu_{2}\right)}, \ldots, \\
& \left.\left.1+\frac{v}{\Gamma\left(3-\mu_{m}\right)}\right)\left\|k_{1}\right\|_{1}\right)<v, \\
& \frac{1}{\Gamma(\beta-2)}\left(\Lambda_{2}+h_{2}\left(1+v, 1+v, 1+\gamma_{0}^{2} v\right. \text {, }\right. \\
& 1+\lambda_{0}^{2} v, 1+\frac{v}{\Gamma(3-q)}, \\
& 1+\frac{v}{\Gamma\left(3-v_{1}\right)}, \\
& 1+\frac{v}{\Gamma\left(3-v_{2}\right)}, \ldots, \\
& \left.\left.1+\frac{v}{\Gamma\left(3-v_{m}\right)}\right)\left\|k_{2}\right\|_{1}\right)<v
\end{aligned}
$$

for all $v \geq L$. Consequently, $\left\|\left(u_{n}, v_{n}\right)\right\|_{* *}<L$ for all $n \geq 1$ and so $\left\{\left(u_{n}, v_{n}\right)\right\}_{n \geq 1}$ is a bounded sequence in $X \times X$. It remains to prove that $\left\{\left(u_{n}, v_{n}\right)^{\prime \prime}\right\}$ is equicontinuous on $[0,1]$. Put

$$
\begin{aligned}
V_{1}=h_{1}\left(1+L, 1+L, 1+\gamma_{0}^{1} L\right. & \\
1 & +\lambda_{0}^{1} L, 1+\frac{L}{\Gamma(3-p)}, 1+\frac{L}{\Gamma\left(3-\mu_{1}\right)}, \\
& \left.1+\frac{L}{\Gamma\left(3-\mu_{2}\right)}, \ldots, 1+\frac{L}{\Gamma\left(3-\mu_{m}\right)}\right),
\end{aligned}
$$




$$
\begin{aligned}
& V_{2}=h_{2}\left(1+L, 1+L, 1+\gamma_{0}^{2} L\right. \\
& 1+\lambda_{0}^{2} L, 1+\frac{L}{\Gamma(3-q)}, 1+\frac{L}{\Gamma\left(3-v_{1}\right)}, \\
& \left.1+\frac{L}{\Gamma\left(3-v_{2}\right)}, \ldots, 1+\frac{L}{\Gamma\left(3-v_{m}\right)}\right), \\
& \Phi_{1}(t)=P_{1}\left(M_{1} t^{\alpha-1}, M_{2} t^{\beta-1}\right. \text {, } \\
& M_{1} \int_{0}^{t} \gamma_{1}(t, s) s^{\alpha-1} d s \\
& M_{2} \int_{0}^{t} \lambda_{1}(t, s) s^{\beta-1} d s \\
& \frac{\left(\delta_{1}-2\right) M_{1}}{\Gamma(\alpha-p)} t^{\alpha-p}, \frac{\left(\delta_{2}-2\right) M_{2}}{\Gamma\left(\beta-\mu_{1}\right)} t^{\beta-\mu_{1}}, \\
& \frac{\left(\delta_{2}-2\right) M_{2}}{\Gamma\left(\beta-\mu_{2}\right)} t^{\beta-\mu_{2}}, \ldots, \\
& \left.\frac{\left(\delta_{2}-2\right) M_{2}}{\Gamma\left(\beta-\mu_{m}\right)} t^{\beta-\mu_{m}}\right) \text {, } \\
& \Phi_{2}(t)=P_{2}\left(M_{1} t^{\alpha-1}, M_{2} t^{\beta-1}\right. \text {, } \\
& M_{1} \int_{0}^{t} \gamma_{2}(t, s) s^{\alpha-1} d s \\
& M_{2} \int_{0}^{t} \lambda_{2}(t, s) s^{\beta-1} d s \\
& \frac{\left(\delta_{2}-2\right) M_{2}}{\Gamma(\beta-q)} t^{\beta-q}, \frac{\left(\delta_{1}-2\right) M_{1}}{\Gamma\left(\alpha-v_{1}\right)} t^{\alpha-v_{1}}, \\
& \left.\frac{\left(\delta_{1}-2\right) M_{1}}{\Gamma\left(\alpha-v_{2}\right)} t^{\alpha-v_{2}}, \ldots, \frac{\left(\delta_{1}-2\right) M_{1}}{\Gamma\left(\alpha-v_{m}\right)} t^{\alpha-v_{m}}\right)
\end{aligned}
$$

for all $t \in(0,1]$. Note that, $\Lambda_{j}=\int_{0}^{1} \Phi_{j}(t) d t$ for $j=1,2$ and

$$
\begin{aligned}
& f_{n}^{1}\left(t, u_{n}(t), v_{n}(t),\left(\phi_{1} u_{n}\right)(t),\right.\left(\psi_{1} v_{n}\right)(t), D_{0^{+}}^{p} u_{n}(t), D_{0^{+}}^{\mu_{1}} v_{n}(t), \\
&\left.D_{0^{+}}^{\mu_{2}} v_{n}(t), \ldots, D_{0^{+}}^{\mu_{m}} v_{n}(t)\right) \\
& \leq \Phi_{1}(t)+V_{1} k_{1}(t), \\
& f_{n}^{2}\left(t, u_{n}(t), v_{n}(t),\left(\phi_{2} u_{n}\right)(t),\right. \\
&\left(\psi_{2} v_{n}\right)(t), D_{0^{+}}^{q} v_{n}(t), D_{0^{+}}^{\nu_{1}} u_{n}(t), \\
&\left.D_{0^{+}}^{\nu_{2}} u_{n}(t), \ldots, D_{0^{+}}^{v_{m}} u_{n}(t)\right) \\
& \leq \Phi_{2}(t)+V_{2} k_{2}(t)
\end{aligned}
$$

for almost all $t \in[0,1]$ and $n \geq 1$. If $0 \leq t_{1}<t_{2} \leq 1$, then

$$
\begin{aligned}
& \left|u_{n}^{\prime \prime}\left(t_{2}\right)-u_{n}^{\prime \prime}\left(t_{1}\right)\right| \\
& =\mid \int_{0}^{1}\left(\frac{\partial^{2}}{\partial t^{2}} G_{\alpha}\left(t_{2}, s\right)-\frac{\partial^{2}}{\partial t^{2}} G_{\alpha}\left(t_{1}, s\right)\right) \\
& f_{n}^{1}\left(s, u_{n}(s), v_{n}(s),\left(\phi_{1} u_{n}\right)(s),\left(\psi_{1} v_{n}\right)(s),\right. \\
& \left.D_{0^{+}}^{p} u_{n}(s), D_{0^{+}}^{\mu_{1}} v_{n}(s), D_{0^{+}}^{\mu_{2}} v_{n}(s), \ldots, D_{0^{+}}^{\mu_{m}} v_{n}(s)\right) d s \\
& \leq \frac{1}{\Gamma(\alpha-2)}\left[\left(t_{2}^{\alpha-3}-t_{1}^{\alpha-3}\right) \int_{0}^{1}\left(\Phi_{1}(s)+V_{1} k_{1}(s)\right) d s\right. \\
& +\int_{t_{1}}^{t_{2}}\left(t_{2}-s\right)^{\alpha-3}\left(\Phi_{1}(s)+V_{1} k_{1}(s)\right) d s \\
& +\int_{0}^{t_{1}}\left(\left(t_{2}-s\right)^{\alpha-3}-\left(t_{1}-s\right)^{\alpha-3}\right) \\
& \left.\times\left(\Phi_{1}(s)+V_{1} k_{1}(s)\right) d s\right] \\
& \leq \frac{1}{\Gamma(\alpha-2)}\left[\left(t_{2}^{\alpha-3}-t_{1}^{\alpha-3}\right)\left(\Lambda_{1}+V_{1}\left\|k_{1}\right\|_{1}\right)\right. \\
& +\left(t_{2}-t_{1}\right)^{\alpha-3}\left(\Lambda_{1}+V_{1}\left\|k_{1}\right\|_{1}\right) \\
& +\int_{0}^{t_{1}}\left(\left(t_{2}-s\right)^{\alpha-3}-\left(t_{1}-s\right)^{\alpha-3}\right) \\
& \left.\times\left(\Phi_{1}(s)+V_{1} k_{1}(s)\right) d s\right] .
\end{aligned}
$$

Similarly, we have

$$
\begin{aligned}
& \left|v_{n}^{\prime \prime}\left(t_{2}\right)-v_{n}^{\prime \prime}\left(t_{1}\right)\right| \\
& \leq \frac{1}{\Gamma(\beta-2)}\left[\left(t_{2}^{\beta-3}-t_{1}^{\beta-3}\right)\left(\Lambda_{2}+V_{2}\left\|k_{2}\right\|_{1}\right)\right. \\
& +\left(t_{2}-t_{1}\right)^{\beta-3}\left(\Lambda_{2}+V_{2}\left\|k_{2}\right\|_{1}\right) \\
& +\int_{0}^{t_{1}}\left(\left(t_{2}-s\right)^{\beta-3}-\left(t_{1}-s\right)^{\beta-3}\right) \\
& \left.\quad \times\left(\Phi_{2}(s)+V_{2} k_{2}(s)\right) d s\right] .
\end{aligned}
$$

Let $\epsilon>0$ be given. Choose $\delta_{0}>0$ such that $0<t_{2}^{\alpha-3}-t_{1}^{\alpha-3}<\epsilon$, $0<t_{2}^{\beta-3}-t_{1}^{\beta-3}<\epsilon$

$$
0<\left(t_{2}-s\right)^{\alpha-3}-\left(t_{1}-s\right)^{\alpha-3}<\epsilon
$$

and $0<\left(t_{2}-s\right)^{\beta-3}-\left(t_{1}-s\right)^{\beta-3}<\epsilon$ for all $0 \leq t_{1}<t_{2} \leq 1$, $t_{2}-t_{1}<\delta_{0}$ and $0 \leq s \leq t_{1}$. Thus, for each $0 \leq t_{1}<t_{2} \leq 1$ and $t_{2}-t_{1}<\min \left\{\delta_{0}, \sqrt[\alpha-3]{\epsilon}, \sqrt[\beta-3]{\epsilon}\right\}$, we have $\left|u_{n}^{\prime \prime}\left(t_{2}\right)-u_{n}^{\prime \prime}\left(t_{1}\right)\right| \leq$ $(3 \epsilon / \Gamma(\alpha-2))\left(\Lambda_{1}+V_{1}\left\|k_{1}\right\|_{1}\right)$ and $\left|v_{n}^{\prime \prime}\left(t_{2}\right)-v_{n}^{\prime \prime}\left(t_{1}\right)\right| \leq(3 \epsilon / \Gamma(\beta-$ 2)) $\left(\Lambda_{2}+V_{2}\left\|k_{2}\right\|_{1}\right)$. Therefore, $\left\{\left(u_{n}, v_{n}\right)^{\prime \prime}\right\}_{n \geq 1}$ is equicontinuous on $[0,1]$. This completes the proof. 
Now, we give our main result.

Theorem 6. The system (1), (2), and (3) has a positive solution $(u, v)$ such that $u(t) \geq M_{1} t^{\alpha-1}, v(t) \geq M_{2} t^{\beta-1}, D_{0^{+}}^{p} u(t) \geq$ $\left(\left(\delta_{1}-2\right) M_{1} / \Gamma(\alpha-p)\right) t^{\alpha-p}, D_{0^{+}}^{q} v(t) \geq\left(\left(\delta_{2}-2\right) M_{2} / \Gamma(\beta-q)\right) t^{\beta-q}$, $D_{0^{+}}^{v_{i}} u(t) \geq\left(\left(\delta_{1}-2\right) M_{1} / \Gamma\left(\alpha-v_{i}\right)\right) t^{\alpha-v_{i}}$, and $D_{0^{+}}^{\mu_{i}} v(t) \geq\left(\left(\delta_{2}-\right.\right.$ 2) $\left.M_{2} / \Gamma\left(\beta-\mu_{i}\right)\right) t^{\beta-\mu_{i}}$ for $i=1,2, \ldots, m$ and $t \in[0,1]$ and $\left(\phi_{j} u\right)(t) \geq M_{1} \int_{0}^{t} \gamma_{j}(t, s) s^{\alpha-1} d s$ and

$$
\left(\psi_{j} v\right)(t) \geq M_{2} \int_{0}^{t} \lambda_{j}(t, s) s^{\beta-1} d s
$$

for $j=1,2$ and $t \in[0,1]$.

Proof. By using Theorem 4, for each natural number $n$, the system (15), (2), and (3) has a solution $\left(u_{n}, v_{n}\right)$ in $P$. Also by using Lemma 5 , the set $\left\{\left(u_{n}, v_{n}\right)\right\}_{n \geq 1}$ is a relatively compact subset of $X \times X$. By using the Arzela-Ascoli theorem, without loss of generality we can assume that $\left\{\left(u_{n}, v_{n}\right)\right\}_{n \geq 1}$ is convergent in $X \times X$ to some element $(u, v)$ of $P$. It is easy to check that $(u, v)$ satisfy the boundary conditions (2) and (3) and also $\lim _{n \rightarrow \infty} D_{0^{+}}^{p} u_{n}=D_{0^{+}}^{p} u, \lim _{n \rightarrow \infty} D_{0^{+}}^{q} v_{n}=D_{0^{+}}^{q} v$, $\lim _{n \rightarrow \infty} D_{0^{+}}^{v_{i}} u_{n}=D_{0^{+}}^{v_{i}} u$, and $\lim _{n \rightarrow \infty} D_{0^{+}}^{\mu_{i}} v_{n}=D_{0^{+}}^{\mu_{i}} v$ for $i=$ $1,2, \ldots, m$ and $\lim _{n \rightarrow \infty} \phi_{j} u_{n}=\phi_{j} u$ and $\lim _{n \rightarrow \infty} \psi_{j} v_{n}=\psi_{j} v$ for $j=1,2$. Thus, it is easy to see that $(u, v)$ satisfy the desired conditions. Also,

$$
\begin{gathered}
\lim _{n \rightarrow \infty} f_{n}^{1}\left(t, u_{n}(t), v_{n}(t),\left(\phi_{1} u_{n}\right)(t),\right. \\
\left(\psi_{1} v_{n}\right)(t), D_{0^{+}}^{p} u_{n}(t), D_{0^{+}}^{\mu_{1}} v_{n}(t), \\
\left.D_{0^{+}}^{\mu_{2}} v_{n}(t), \ldots, D_{0^{+}}^{\mu_{m}} v_{n}(t)\right) \\
=f_{1}\left(t, u(t), v(t),\left(\phi_{1} u\right)(t),\left(\psi_{1} v\right)(t),\right. \\
D_{0^{+}}^{p} u(t), D_{0^{+}}^{\mu_{1}} v(t) \\
\left.D_{0^{+}}^{\mu_{2}} v(t), \ldots, D_{0^{+}}^{\mu_{m}} v(t)\right) \\
\lim _{n \rightarrow \infty}^{2}\left(t, u_{n}(t), v_{n}(t),\left(\phi_{2} u_{n}\right)(t)\right. \\
\left(\psi_{2} v_{n}\right)(t), D_{0^{+}}^{q} v_{n}(t), D_{0^{+}}^{\nu_{1}} u_{n}(t), \\
\left.D_{0^{+}}^{\nu_{2}} u_{n}(t), \ldots, D_{0^{+}}^{\nu_{m}} u_{n}(t)\right) \\
=f_{2}\left(t, u(t), v(t),\left(\phi_{2} u\right)(t)\right. \\
\left(\psi_{2} v\right)(t), D_{0^{+}}^{q} v(t), D_{0^{+}}^{\nu_{1}} u(t) \\
\left.D_{0^{+}}^{\nu_{2}} u(t), \ldots, D_{0^{+}}^{\nu_{m}} u(t)\right)
\end{gathered}
$$

for almost all $t \in[0,1]$. If $\xi=\sup \left\{\left\|\left(u_{n}, v_{n}\right)\right\|_{* *}: n \geq 1\right\}$, then $\left\|D_{0^{+}}^{p} u_{n}\right\| \leq \xi / \Gamma(3-p),\left\|D_{0^{+}}^{q} v_{n}\right\| \leq \xi / \Gamma(3-q),\left\|D_{0^{+}}^{v_{i}} u_{n}\right\| \leq$ $\xi / \Gamma\left(3-v_{i}\right)$, and $\left\|D_{0^{+}}^{\mu_{i}} v_{n}\right\| \leq \xi / \Gamma\left(3-\mu_{i}\right)$ for $i=1,2, \ldots, m$ and $n \geq 1\left\|\phi_{j} u_{n}\right\| \leq \xi \gamma_{0}^{j}$ and $\left\|\psi_{j} v_{n}\right\| \leq \xi \lambda_{0}^{j}$ for $j=1,2$ and $n \geq 1$. Thus,

$$
\begin{aligned}
& 0 \leq G_{\alpha}(t, s) f_{n}^{1}\left(s, u_{n}(s), v_{n}(s),\right. \\
& \left(\phi_{1} u_{n}\right)(s),\left(\psi_{1} v_{n}\right)(s), \\
& D_{0^{+}}^{p} u_{n}(s), D_{0^{+}}^{\mu_{1}} v_{n}(s), \\
& \left.D_{0^{+}}^{\mu_{2}} v_{n}(s), \ldots, D_{0^{+}}^{\mu_{m}} v_{n}(s)\right) \\
& \leq \frac{1}{\Gamma(\alpha)}\left(\Phi_{1}(s)+h_{1}(1+\xi,\right. \\
& 1+\xi, 1+\gamma_{0}^{1} \xi, 1+\lambda_{0}^{1} \xi \\
& 1+\frac{\xi}{\Gamma(3-p)}, 1+\frac{\xi}{\Gamma\left(3-\mu_{1}\right)}, \\
& 1+\frac{\xi}{\Gamma\left(3-\mu_{2}\right)}, \ldots, \\
& \left.\left.1+\frac{\xi}{\Gamma\left(3-\mu_{m}\right)}\right) k_{1}(s)\right), \\
& 0 \leq G_{\beta}(t, s) f_{n}^{2}\left(s, u_{n}(s), v_{n}(s),\left(\phi_{2} u_{n}\right)(s)\right. \text {, } \\
& \left(\psi_{2} v_{n}\right)(s), D_{0^{+}}^{q} v_{n}(s), D_{0^{+}}^{v_{1}} u_{n}(s), \\
& \left.D_{0^{+}}^{v_{2}} u_{n}(s), \ldots, D_{0^{+}}^{v_{m}} u_{n}(s)\right) \\
& \leq \frac{1}{\Gamma(\beta)}\left(\Phi_{2}(s)+h_{2}(1+\xi,\right. \\
& 1+\xi, 1+\gamma_{0}^{2} \xi, 1+\lambda_{0}^{2} \xi \\
& 1+\frac{\xi}{\Gamma(3-q)}, 1+\frac{\xi}{\Gamma\left(3-v_{1}\right)}, \\
& 1+\frac{\xi}{\Gamma\left(3-v_{2}\right)}, \ldots, \\
& \left.\left.1+\frac{\xi}{\Gamma\left(3-v_{m}\right)}\right) k_{2}(s)\right)
\end{aligned}
$$

for almost all $(t, s) \in[0,1] \times[0,1]$ and $n \geq 1$. Hence, by using the Lebesgue dominated convergence theorem we get

$$
\begin{gathered}
u(t) \\
=\int_{0}^{1} G_{\alpha}(t, s) f_{1}\left(s, u(s), v(s),\left(\phi_{1} u\right)(s),\left(\psi_{1} v\right)(s),\right. \\
D_{0^{+}}^{p} u(s), D_{0^{+}}^{\mu_{1}} v(s), D_{0^{+}}^{\mu_{2}} v(s), \ldots, \\
\left.D_{0^{+}}^{\mu_{m}} v(s)\right) d s,
\end{gathered}
$$


$v(t)$

$$
\begin{aligned}
=\int_{0}^{1} G_{\beta}(t, s) f_{2}\left(s, u(s), v(s),\left(\phi_{2} u\right)(s),\right. \\
\left(\psi_{2} v\right)(s), D_{0^{+}}^{q} v(s), D_{0^{+}}^{\nu_{1}} u(s), \\
\left.D_{0^{+}}^{\nu_{2}} u(s), \ldots, D_{0^{+}}^{\nu_{m}} u(s)\right) d s
\end{aligned}
$$

for all $t \in[0,1]$. Therefore, $(u, v)$ is a positive solution of the system (1), (2), and (3).

\section{Example}

Here, we give an example to illustrate our last result.

Example 1. Let $m_{1}>0, m_{2}>0, n \geq 4, n-1<\alpha, \beta<n$, $0<p, q<1,1<\mu_{i}, v_{i}<2$ for $i=1,2, \ldots, m, \rho_{1}, \rho_{2}, \sigma_{1}, \sigma_{2} \in$ $L^{1}[0,1], \sigma_{1}(t) \geq m_{1}$ and $\sigma_{2}(t) \geq m_{2}$ for almost all $t \in[0,1]$. Suppose that $a_{1}, c_{1} \in(0,1 /(\alpha-1)), a_{2}, c_{2} \in(0,1 /(\beta-1))$, $a_{5} \in(0,1 /(\alpha-p)), c_{5} \in(0,1 /(\beta-q)), a_{i}^{\prime} \in\left(0,1 /\left(\beta-\mu_{i}\right)\right)$, and $c_{i}^{\prime} \in\left(0,1 /\left(\alpha-v_{i}\right)\right)$ for $i=1,2, \ldots, m$ and also $b_{1}$, $b_{2}, b_{3}, b_{4}, b_{5}, b_{1}^{\prime}, b_{2}^{\prime}, \ldots, b_{m}^{\prime}, d_{1}, d_{2}, d_{3}, d_{4}, d_{5}, d_{1}^{\prime}, d_{2}^{\prime}, \ldots, d_{m}^{\prime} \in(0$, $1)$. Assume that $a_{3}, a_{4}, c_{3}$ and $c_{4}$ are positive real numbers such that $\int_{0}^{1}\left(\int_{0}^{s} \gamma_{1}(s, \tau) \tau^{\alpha-1} d \tau\right)^{-a_{3}} d s<\infty, \int_{0}^{1}\left(\int_{0}^{s} \gamma_{2}(s\right.$, $\left.\tau) \tau^{\alpha-1} d \tau\right)^{-c_{3}} d s<\infty, \int_{0}^{1}\left(\int_{0}^{s} \lambda_{1}(s, \tau) \tau^{\beta-1} d \tau\right)^{-a_{4}} d s<\infty$, and $\int_{0}^{1}\left(\int_{0}^{s} \lambda_{2}(s, \tau) \tau^{\beta-1} d \tau\right)^{-c_{4}} d s<\infty$. Define the functions $f_{1}$ and $f_{2}$ on $[0,1] \times \mathfrak{D}$ by

$$
\begin{gathered}
f_{1}\left(t, x, y, z, w, v, u_{1}, u_{2}, \ldots, u_{m}\right) \\
=\frac{1}{x^{a_{1}}}+\frac{1}{y^{a_{2}}}+\frac{1}{z^{a_{3}}}+\frac{1}{w^{a_{4}}} \\
+\frac{1}{v^{a_{5}}}+\frac{1}{u_{1}^{a_{1}^{\prime}}}+\frac{1}{u_{2}^{a_{2}^{\prime}}}+\cdots+\frac{1}{u_{m}^{a_{m}^{\prime}}} \\
+\left|\rho_{1}(t)\right|\left(x^{b_{1}}+y^{b_{2}}+z^{b_{3}}\right. \\
\quad+w^{b_{4}}+v^{b_{5}}+u_{1}^{b_{1}^{\prime}} \\
\left.+u_{2}^{b_{2}^{\prime}}+\cdots+u_{m}^{b_{m}^{\prime}}\right)+\sigma_{1}(t), \\
f_{2}\left(t, x, y, z, w, v, u_{1}, u_{2}, \ldots, u_{m}\right) \\
=\frac{1}{x^{c_{1}}}+\frac{1}{y^{c_{2}}}+\frac{1}{z^{c_{3}}}+\frac{1}{w^{c_{4}}} \\
+\frac{1}{v^{c_{5}}}+\frac{1}{u_{1}^{c_{1}^{\prime}}}+\frac{1}{u_{2}^{c_{2}^{\prime}}}+\cdots+\frac{1}{u_{m}^{c_{m}^{\prime}}}
\end{gathered}
$$

$$
\begin{aligned}
+\left|\rho_{2}(t)\right| & \left(x^{d_{1}}+y^{d_{2}}+z^{d_{3}}\right. \\
& +w^{d_{4}}+v^{d_{5}}+u_{1}^{d_{1}^{\prime}} \\
& \left.+u_{2}^{d_{2}^{\prime}}+\cdots+u_{m}^{d_{m}^{\prime}}\right)+\sigma_{2}(t) .
\end{aligned}
$$

Note that the functions $f_{1}$ and $f_{2}$ satisfy the conditions $\left(H_{1}\right)$ and $\left(\mathrm{H}_{2}\right)$, where

$$
\begin{gathered}
P_{1}\left(x, y, z, w, v, u_{1}, u_{2}, \ldots, u_{m}\right) \\
=\frac{1}{x^{a_{1}}}+\frac{1}{y^{a_{2}}}+\frac{1}{z^{a_{3}}}+\frac{1}{w^{a_{4}}} \\
+\frac{1}{v^{a_{5}}}+\frac{1}{u_{1}^{a_{1}^{\prime}}}+\frac{1}{u_{2}^{a_{2}^{\prime}}}+\cdots+\frac{1}{u_{m}^{a_{m}^{\prime}}}, \\
P_{2}\left(x, y, z, w, v, u_{1}, u_{2}, \ldots, u_{m}\right) \\
=\frac{1}{x^{c_{1}}}+\frac{1}{y^{c_{2}}}+\frac{1}{z^{c_{3}}}+\frac{1}{w^{c_{4}}} \\
\quad+\frac{1}{v^{c_{5}}}+\frac{1}{u_{1}^{c_{1}^{\prime}}}+\frac{1}{u_{2}^{c_{2}^{\prime}}}+\cdots+\frac{1}{u_{m}^{c_{m}^{\prime}}} \\
h_{1}\left(x, y, z, w, v, u_{1}, u_{2}, \ldots, u_{m}\right) \\
=1+x^{b_{1}}+y^{b_{2}}+z^{b_{3}}+w^{b_{4}} \\
\quad+v^{b_{5}}+u_{1}^{b_{1}^{\prime}}+u_{2}^{b_{2}^{\prime}}+\cdots+u_{m}^{b_{m}^{\prime}}, \\
h_{2}\left(x, y, z, w, v, u_{1}, u_{2}, \ldots, u_{m}\right) \\
=1+x^{d_{5}}+u_{1}^{d_{1}^{\prime}}+u_{2}^{d_{2}^{\prime}}+\cdots+u_{m}^{d_{m}^{\prime}},
\end{gathered}
$$

$k_{1}(t)=\left|\rho_{1}(t)\right|+\sigma_{1}(t)$ and $k_{2}(t)=\left|\rho_{2}(t)\right|+\sigma_{2}(t)$. Theorem 6 guarantees that the system (1), (2), and (3) via these functions has a positive solution $(u, v)$ satisfying the desired inequalities in Theorem 6 whenever $M_{1}=m_{1} /\left(\alpha-\delta_{1}\right) \Gamma(\alpha+1)$ and $M_{2}=$ $m_{2} /\left(\beta-\delta_{2}\right) \Gamma(\beta+1)$.

\section{Conclusions}

One of the most interesting branches is obtaining solutions of singular fractional differential equations via boundary value problems. Having these thought in mind we discuss the existence of positive solutions for a coupled system of multiterm singular fractional integrodifferential boundary value problems. An illustrative example illustrates the applicability of the proposed method.

\section{Conflict of Interests}

The authors declare that there is no conflict of interests regarding the publication of this paper. 


\section{Acknowledgments}

The research of the second and third authors was supported by Azarbaijan Shahid Madani University. Also, the authors express their gratitude to the referees for their helpful suggestions which improved the final version of this paper.

\section{References}

[1] R. P. Agarwal, D. O’Regan, and S. Staněk, "Positive solutions for Dirichlet problems of singular nonlinear fractional differential equations," Journal of Mathematical Analysis and Applications, vol. 371, no. 1, pp. 57-68, 2010.

[2] R. P. Agarwal, D. O’Regan, and S. Staněk, "Positive solutions for mixed problems of singular fractional differential equations," Mathematische Nachrichten, vol. 285, no. 1, pp. 27-41, 2012.

[3] R. P. Agarwal, Y. Zhou, and Y. He, "Existence of fractional neutral functional differential equations," Computers \& Mathematics with Applications, vol. 59, no. 3, pp. 1095-1100, 2010.

[4] B. Ahmad and A. Alsaedi, "Existence and uniqueness of solutions for coupled systems of higher-order nonlinear fractional differential equations," Fixed Point Theory and Applications, vol. 2010, Article ID 364560, 17 pages, 2010.

[5] B. Ahmad and J. J. Nieto, "Existence results for a coupled system of nonlinear fractional differential equations with threepoint boundary conditions," Computers \& Mathematics with Applications, vol. 58, no. 9, pp. 1838-1843, 2009.

[6] C.-Z. Bai and J.-X. Fang, "The existence of a positive solution for a singular coupled system of nonlinear fractional differential equations," Applied Mathematics and Computation, vol. 150, no. 3, pp. 611-621, 2004.

[7] Z. Bai and H. Lü, "Positive solutions for boundary value problem of nonlinear fractional differential equation," Journal of Mathematical Analysis and Applications, vol. 311, no. 2, pp. 495-505, 2005.

[8] Z. Bai and W. Sun, "Existence and multiplicity of positive solutions for singular fractional boundary value problems," Computers \& Mathematics with Applications, vol. 63, no. 9, pp. 1369-1381, 2012.

[9] D. Baleanu, R. P. Agarwal, H. Mohammadi, and S. Rezapour, "Some existence results for a nonlinear fractional differential equation on partially ordered Banach spaces," Boundary Value Problems, vol. 2013, article 112, 2013.

[10] D. Baleanu, H. Mohammadi, and Sh. Rezapour, "Positive solutions of an initial value problem for nonlinear fractional differential equations," Abstract and Applied Analysis, vol. 2012, Article ID 837437, 7 pages, 2012.

[11] D. Baleanu, S. Rezapour, and H. Mohammadi, "Some existence results on nonlinear fractional differential equations," Philosophical Transactions of the Royal Society of London A, vol. 371, no. 1990, Article ID 20120144, 7 pages, 2013.

[12] D. Baleanu, H. Mohammadi, and S. Rezapour, "On a nonlinear fractional differential equation on partially ordered metric spaces," Advances in Difference Equations, vol. 2013, article 83, 2013.

[13] M. Benchohra, S. Hamani, and S. K. Ntouyas, "Boundary value problems for differential equations with fractional order and nonlocal conditions," Nonlinear Analysis: Theory, Methods \& Applications, vol. 71, no. 7-8, pp. 2391-2396, 2009.

[14] Y.-K. Chang and J. J. Nieto, "Some new existence results for fractional differential inclusions with boundary conditions,"
Mathematical and Computer Modelling, vol. 49, no. 3-4, pp. 605$609,2009$.

[15] F. Chen and Y. Zhou, "Attractivity of fractional functional differential equations," Computers \& Mathematics with Applications, vol. 62, no. 3, pp. 1359-1369, 2011.

[16] W. Feng, S. Sun, Z. Han, and Y. Zhao, "Existence of solutions for a singular system of nonlinear fractional differential equations," Computers \& Mathematics with Applications, vol. 62, no. 3, pp. 1370-1378, 2011.

[17] Y. Liu, w. Zhang, and X. Liu, "A sufficient condition for the existence of a positive solution for a nonlinear fractional differential equation with the Riemann-Liouville derivative," Applied Mathematics Letters, vol. 25, no. 11, pp. 1986-1992, 2012.

[18] I. Podlubny, Fractional Differential Equations, vol. 198 of Mathematics in Science and Engineering, Academic Press, San Diego, Calif, USA, 1999.

[19] S. Staněk, "The existence of positive solutions of singular fractional boundary value problems," Computers \& Mathematics with Applications, vol. 62, no. 3, pp. 1379-1388, 2011.

[20] X. Su, "Boundary value problem for a coupled system of nonlinear fractional differential equations," Applied Mathematics Letters, vol. 22, no. 1, pp. 64-69, 2009.

[21] S. Sun, Q. Li, and Y. Li, "Existence and uniqueness of solutions for a coupled system of multi-term nonlinear fractional differential equations," Computers \& Mathematics with Applications, vol. 64, no. 10, pp. 3310-3320, 2012. 


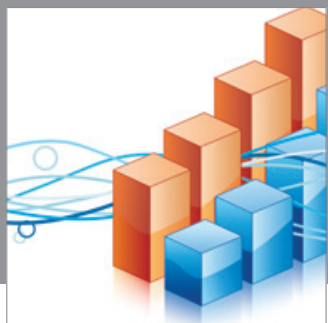

Advances in

Operations Research

mansans

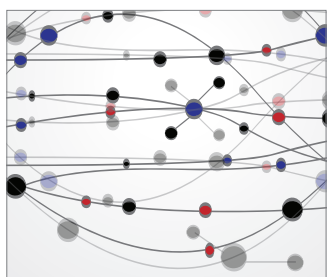

The Scientific World Journal
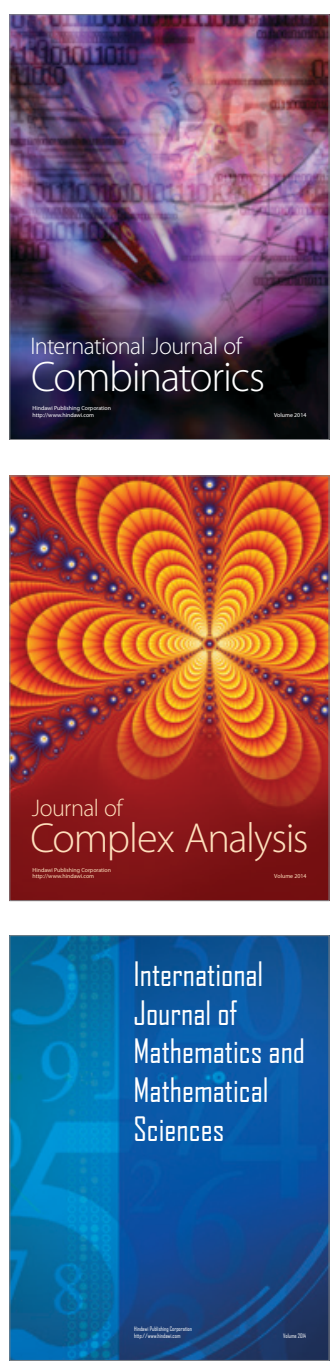
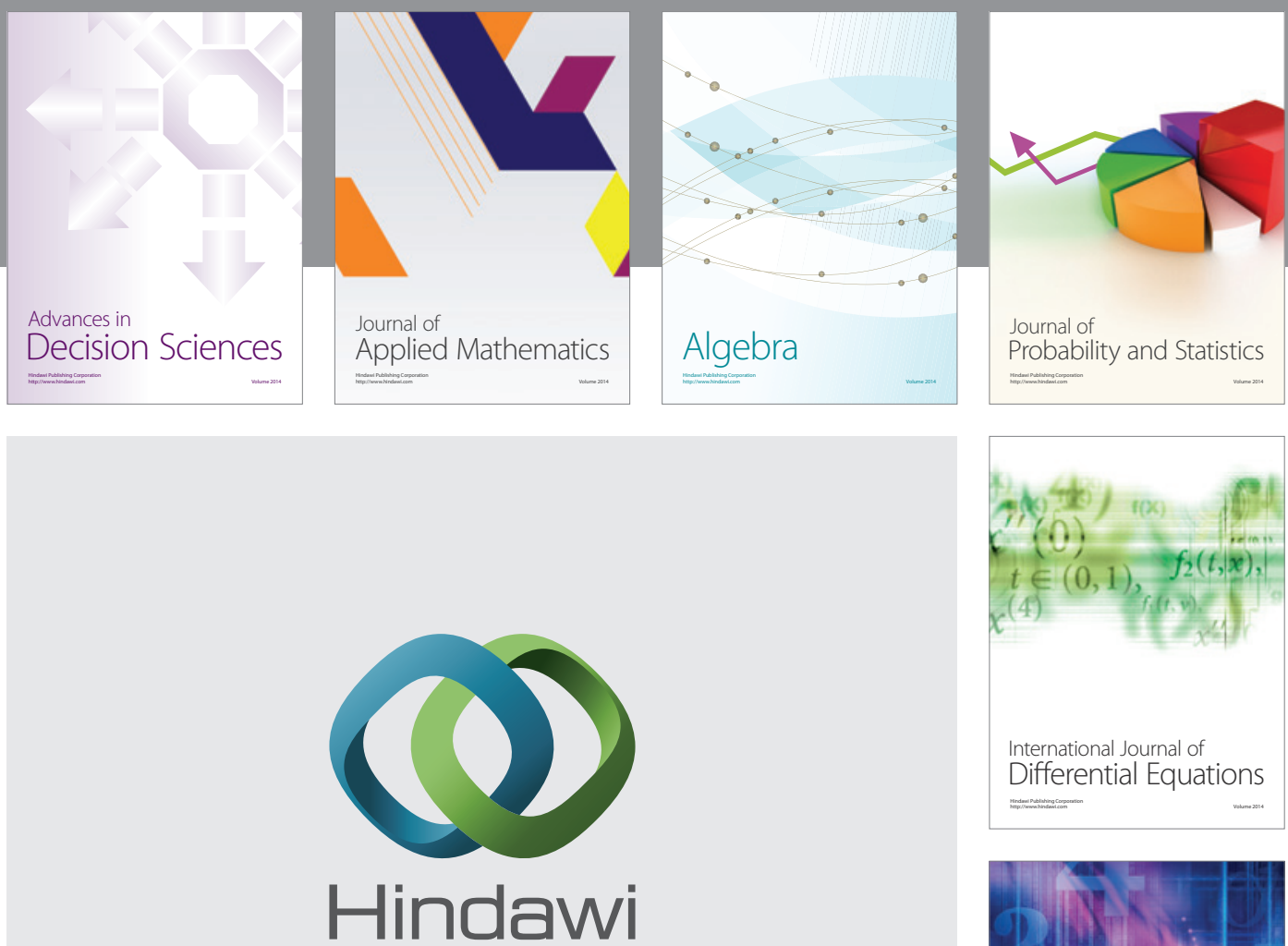

Submit your manuscripts at http://www.hindawi.com
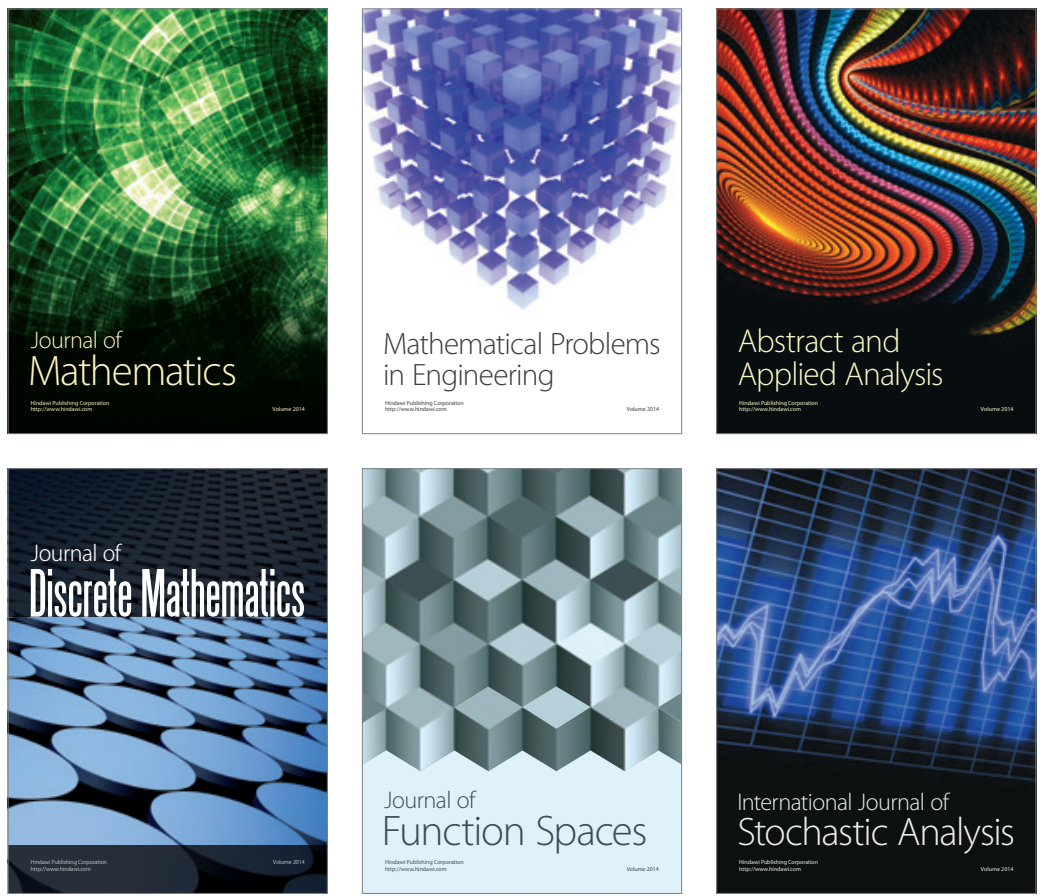

Journal of

Function Spaces

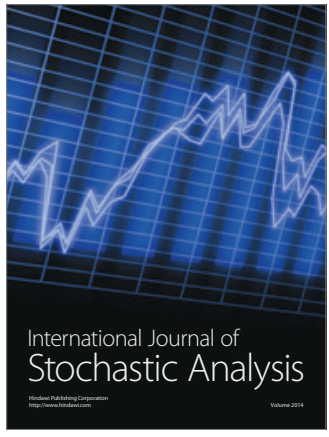

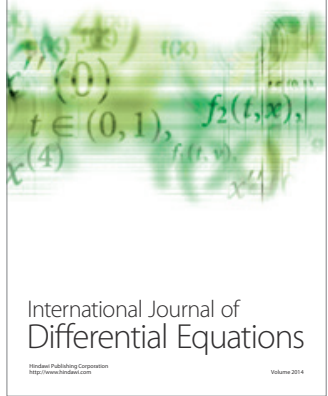
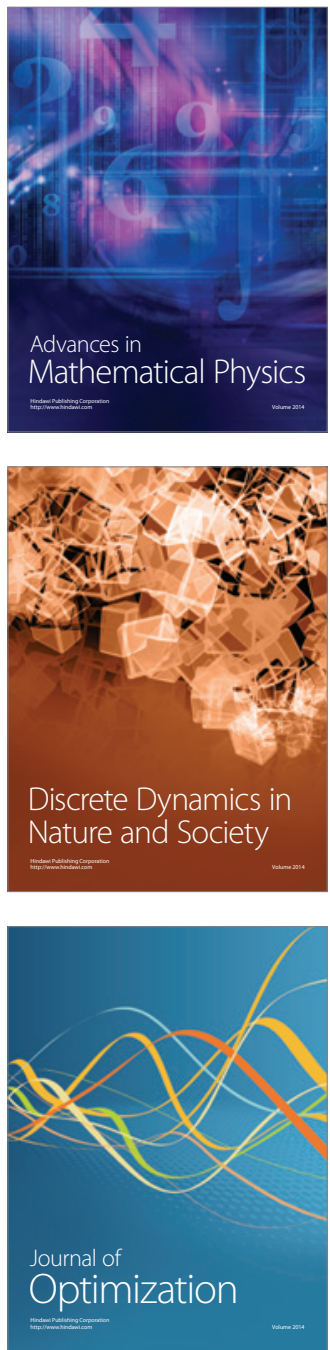